\begin{tabular}{|c|l|}
\hline Title & Existence and non-existence results for wave operators for perturbations of the laplacian \\
\hline Author(s) & Jensen, A.; Ozawa, T. \\
\hline Citation & Hokkaido University Preprint Series in Mathematics, 188, 1-30 \\
\hline Issue Date & 1993-03 \\
\hline DOI & 10.14943/83332 \\
\hline Doc URL & http://hdl.handle.net/2115/68934 \\
\hline Type & bulletin (article) \\
\hline File Information & pre188.pdf \\
\hline
\end{tabular}

Instructions for use 
EXISTENCE AND NON-EXISTENCE

RESULTS FOR WAVE OPERATORS FOR PERTURBATIONS OF THE LAPLACIAN

A. Jensen and T. Ozawa

Series $\sharp 188$. March 1993 


\section{HOKKAIDO UNIVERSITY \\ PREPRINT SERIES IN MATHEMATICS}

\#160: A. Arai, Properties of the Dirac-Weyl operator with a strongly singular gauge potential, 26 pages. 1992.

$\sharp 161$ : A. Arai, Dirac operators in Boson-Fermion Fock spaces and supersymmetric quantum field theory, 30 pages. 1992.

$\sharp 162$ : S. Albeverio, K. Iwata, T. Kolsrud, Random parallel transport on surfaces of finite type, and relations to homotopy, 8 pages. 1992.

\#163: S. Albeverio, K. Iwata, T. Kolsrud, Moments of random fields over a family of elliptic curves, and modular forms, 9 pages. 1992.

\#164: Y. Giga, M. Sato, Neumann problem for singular degenerate parabolic equations, 12 pages. 1992.

\#165: J. Wierzbicki, Y. Watatani, Commuting squares and relative entropy for two subfactors, 18 pages. 1992.

$\sharp 166$ : Y. Okabe, A new algorithm driven from the view-point of the fluctuation-dissipation theorem in the theory of $\mathrm{KM}_{2} \mathrm{O}$-Langevin equations, 13 pages. 1992.

$\# 167$ : Y. Okabe, H. Mano and Y. Itoh, Random collision model for interacting populations of two species and its strong law of large numbers, 14 pages. 1992.

$\sharp 168$ : A. Inoue, On the equations of stationary precesses with divergent diffusion coefficients, 25 pages. 1992.

\#169: T. Ozawa, Remarks on quadratic nonlinear Schrödinger equations, 19 pages. 1992.

$\$ 170: \quad$ T. Fukui, Y. Giga, Motion of a graph by nonsmooth weighted curvature, 11 pages. 1992.

$\sharp 171$ : J. Inoue, T. Nakazi, Finite dimensional solution sets of extremal problems in $\mathrm{H}^{1}, 10$ pages. 1992.

$\sharp 172$ : S. Izumiya, A characterization of complete integrability for partial differential equations of first order, 6 pages. 1992.

$\sharp 173$ : T. Suwa, Unfoldings of codimension one complex analytic foliation singularities, 49 pages. 1992.

$\sharp 174$ : T. Ozawa, Wave propagation in even dimensional spaces, 15 pages. 1992.

\#175: S. Izumiya, Systems of Clairaut type, 7 pages. 1992.

$\sharp 176$ : A. Hoshiga, The initial value problems for quasi-linear wave equations in two space dimensions with small data, 25 pages. 1992.

$\sharp 177$ : $\quad$ K. Sugano, On bicommutators of modules over H-separable extension rings III, 9 pages. 1993.

\#178: T. Nakazi, Toeplitz operators and weighted norm inequalities, 17 pages. 1993.

$\sharp 179: \quad$ O. Ogurisu, Existence and structure of infinitely degenerate zero-energy ground states of a Wess-Zumino type model in supersymmetric quantum mechanics, 26 pages. 1993.

$\sharp 180$ : O. Ogurisu, Ground state of a spin 1/2 charged particle in an even dimensional magnetic field, 9 pages. 1993.

$\sharp 181$ : K. Sugano, Note on H-separable Galois extension, 6 pages. 1993.

\#182: M. Yamada, Distance formulas of asymptotic Toeplitz and Hankel operators, 13 pages. 1993

$\sharp 183: \quad$ G. Ishikawa, T. Ozawa, The genus of a connected compact real algebraic surface in the affine three space, 11 pages. 1993.

$\sharp 184$ : T. Hibi, Canonical modules and Cohen-Macaulay types of partially ordered sets, 6 pages. 1993.

$\$ 185$ : Y. Giga, K. Yama-uchi, On a lower bound for the extinction time of surfaces moved by mean curvature, 16 pages. 1993.

\# 186: Y. Kurokawa, On functional moduli for first order ordinary differential equations, 9 pages. 1993.

$\# 187$ : O. Ogurisu, Unitary equivalence between a spin $1 / 2$ charged particle in a two-dimensional magnetic field and a spin $1 / 2$ neutral particle with an anomalons magnetic moment in a two-dimensional electric field, 4 pages. 


\title{
EXISTENCE AND NON-EXISTENCE RESULTS FOR WAVE OPERATORS FOR PERTURBATIONS OF THE LAPLACIAN
}

\author{
ARNE JENSEN AND TOHRU OZAWA
}

\begin{abstract}
Schrödinger operators with time-dependent potentials are studied. Necessary and sufficient conditions for existence of ordinary and Dollard-type modified wave operators are obtained. Sharp results for potentials with a specified leading term are obtained. Applications are given to the surfboard Schrödinger equation and to Stark Hamiltonians. In the latter case the discrepancy between classical and quantum scattering in dimension one is resolved.
\end{abstract}

\section{INTRODUCTION}

Let $H(t)=-\frac{1}{2} \Delta+V(t, x)$ be a time-dependent Schrödinger operator. The associated unitary propagator is denoted $U(t, s)$. We give a series of necessary and sufficient conditions on $V(t, x)$ for the existence of the ordinary wave operators $W_{ \pm}=\mathrm{s}-\lim _{t \rightarrow \pm \infty} U(0, t) U_{0}(t)$ and the Dollard-type modified wave operators $W_{ \pm}^{D}=$ $\mathrm{s}-\lim _{t \rightarrow \pm \infty} U(0, t) U_{D}(t)$, where $U_{0}(t)=\exp \left(\frac{i t}{2} \Delta\right)$ is the free evolution and $U_{D}(t)=$ $U_{0}(t) \exp \left(-i \int_{0}^{t} V_{\ell}(\tau,-i \tau \nabla) d \tau\right)$ is the Dollard modified free evolution, with a decomposition into short range and long range parts $V=V_{s}+V_{l}$.

The results are applied to Stark Hamiltonians and to the surfboard Schrödinger equation, which fit into the present framework in the moving frame picture. In particular, the results obtained here resolve the discrepancy between classical and quantum scattering for one dimensional Stark Hamiltonians discovered in [8]. This is done by proving existence of the quantum wave operators with the time-dependent pure phase modifier proposed by Graf [5] for potentials satisfying $|V(x)|+\left|V^{\prime}(x)\right| \leq c(\log |x|)^{-\beta}$, $|x|>2, \beta>1$. This is the same class of potentials under which classical scattering is studied in [8]. For the surfboard equations we prove existence and unitarity of the modified wave operators for potentials with minimal regularity, generalizing some results in [22].

The main idea in the proofs given here is an effective use of the well-known factorization of the free evolution $U_{0}(t)=M(t) D(t) F M(t)$ where $M(t)=\exp \left(i x^{2} / 2 t\right)$ is a multiplication operator, $F$ the Fourier transform, and $D(t)$ the modified dilation given by $(D(t) \varphi)(x)=(i t)^{-n / 2} \varphi\left(t^{-1} x\right)$. This factorization makes obvious the $t^{-1} x$ nature of the free evolution. This idea has recently been used in the study of long 
range scattering for non-linear Schrödinger equations, see [13, 4]. Related results using this factorization for proving existence have been given by Yafaev in [20].

No precise statement of our results will be given here, since several definitions are required as preparation. Let us briefly indicate the contents and point out the main theorems. In Section 2 results on existence of Dollard-type modified wave operators are obtained. The main results are Theorem 2.3 for general existence and Theorems 2.13 and 2.16 on Stark Hamiltonians. In Section 3 a series of nonexistence results are obtained for ordinary wave operators (Theorem 3.2), Dollard modified wave operators (Theorem 3.10) and for Stark Hamiltonians (Theorem 3.7). Assuming a simple form of the leading behavior of the potential the results show in a very precise manner that the behavior $V(x) \sim|x|^{-1 / 2}$ as $|x| \rightarrow \infty$ is the borderline case for the existence of the Dollard type modified wave operators. See Example 3.11. In Section 4 existence and unitarity is proved for the modified wave operators for the surfboard Schrödinger equation with minimal regularity assumptions on the potential, see Theorem 4.5. Finally, in section 5 the results obtained here are discussed and compared with other results in the literature. In particular, Stark Hamiltonians are discussed in some detail.

Acknowledgement. T. Ozawa thanks Professor Jean Ginibre for suggesting using the factorized free evolution in the study of the surfboard Schrödinger equation as well as for communicating unpublished material on this subject. This research was carried out at the Mittag-Leffler Institute. The support and hospitality of the Institute are gratefully acknowledged.

\section{Existence of Wave Operators}

In this section we give results on the existence of modified wave operators of Dollard type for a class of time-dependent potentials. Obviously the ordinary wave operators are included as the special case where the long range part of the potential vanishes.

2.1. Notation. Assumptions. Let $H_{0}=-\frac{1}{2} \Delta$ on $L^{2}\left(\mathbf{R}^{n}\right)$ with domain $\mathcal{D}\left(H_{0}\right)=$ $H^{2}\left(\mathbf{R}^{n}\right)$, the usual Sobolev space. The potential is a measurable function $V: \mathbf{R} \times$ $\mathbf{R}^{n} \rightarrow \mathbf{R}$. We write $V(t)$ or $V(t, \cdot)$ when we consider the potential as a multiplication operator on $L^{2}\left(\mathbf{R}^{n}\right)$ depending on the parameter $t$, and we write

$$
H(t)=H_{0}+V(t, \cdot)
$$

for the family of time-dependent Hamiltonians. We consider the problem

$$
i \frac{d \varphi}{d t}=H(t) \varphi, \quad \varphi(s)=\varphi_{0} .
$$

The associated propagator is denoted by $U(t, s)$ such that the solution to the problem $(2.2)$ is given by $\varphi(t)=U(t, s) \varphi_{0}$.

The first assumption is 
Assumption 2.1. Let $V: \mathbf{R} \times \mathbf{R}^{n} \rightarrow \mathbf{R}$ be a measurable function. Assume that there exists a unique unitary propagator $U(t, s)$ associated with the problem (2.1) and (2.2).

Throughout this paper we will impose Assumption 2.1 on $V$. Sufficient conditions for $V$ to satisfy Assumption 2.1 can be found in [23].

For the special case $V \equiv 0$ we write $U_{0}(t)=\exp \left(-i t H_{0}\right)$ for the propagator. The following factorization is used throughout the paper

$$
U_{0}(t)=M(t) D(t) F M(t)
$$

Here $M(t)=\exp \left(i x^{2} / 2 t\right)$ as a multiplication operator, $D(t)$ is the modified dilation given by $(D(t) \varphi)(x)=(i t)^{-n / 2} \varphi\left(t^{-1} x\right)$. Furthermore,

$$
(F \varphi)(\xi)=\hat{\varphi}(\xi)=(2 \pi)^{-n / 2} \int e^{-i x \cdot \xi} \varphi(x) d x
$$

denotes the Fourier transform. The inverse Fourier transform of $\varphi$ is denoted $\check{\varphi}$.

For the long range part of the potential $V_{\ell}$ we introduce the function

$$
S(t, y)=-\int_{0}^{t} V_{\ell}(\tau, \tau y) d \tau
$$

We write $S(t)$ or $S(t, \cdot)$ for the multiplication operator and $S(t,-i \nabla)$ for the operator defined via the functional calculus (or using the Fourier transform). These conventions will be used without further comments in the sequel.

For $K \subseteq \mathrm{R}^{n}$ the characteristic function is denoted by $\chi_{K}$. The bounded operators on $L^{2}\left(\mathbf{R}^{n}\right)$ are denoted by $\mathcal{B}\left(L^{2}\left(\mathbf{R}^{n}\right)\right)$. The norm on $L^{p}\left(\mathbf{R}^{n}\right)$ is denoted by $\|\cdot\|_{p}$. The gradient and Laplacian on $\mathbf{R}^{n}$ are denoted by $\nabla$ and by $\Delta$, respectively. We abuse notation and write $\nabla \Phi \in L^{\infty}\left(\mathbf{R}^{n}\right)$ instead of $\nabla \Phi \in\left(L^{\infty}\left(\mathbf{R}^{n}\right)\right)^{n}$.

Throughout the paper $N \subset \mathbf{R}^{n}$ denotes a closed set of measure zero, depending on the potential and its decomposition introduced in the following assumption.

Assumption 2.2. Let $V$ satisfy Assumption 2.1. Assume $V=V_{\ell}+V_{s}$. Assume there is a real number $m \geq 0$ such that

(SR) For any compact $K \subset \mathbf{R}^{n} \backslash N$ the time-dependent operator

$$
\chi_{K}(\cdot) V_{s}(t, t(\cdot))(1-\Delta)^{-m}
$$

is bounded on $L^{2}\left(\mathbf{R}^{n}\right)$ and for some $t_{0} \geq 0$

$$
\int_{|t| \geq t_{0}}\left\|\chi_{K}(\cdot) V_{s}(t, t(\cdot))(1-\Delta)^{-m}\right\|_{\mathcal{B}\left(L^{2}\left(\mathbf{R}^{n}\right)\right)} d t<\infty .
$$


(LR) For any compact $K \subset \mathrm{R}^{n} \backslash N$ there exists $t_{K}>0$ such that $\nabla S(t) \in L^{\infty}(K)$, $\Delta S(t) \in L^{\infty}(K)$ for all $t,|t| \geq t_{K}$, and

$$
\begin{aligned}
& \lim _{|t| \rightarrow \infty}|t|^{-1 / 2}\|\nabla S(t)\|_{L^{\infty}(K)}=0, \\
& \int_{|t| \geq t_{K}} t^{-2}\|\nabla S(t)\|_{L^{\infty}(K)}^{2} d t<\infty, \\
& \int_{|t| \geq t_{K}} t^{-2}\|\Delta S(t)\|_{L^{\infty}(K)} d t<\infty .
\end{aligned}
$$

Let us note that it suffices to check the conditions (SR) and (LR) for a sequence of compact sets $K_{j} \subset \mathrm{R}^{n} \backslash N, j=1,2, \ldots$ with $K_{j} \subset K_{j+1}$ and $\cup_{j \geq 1} K_{j}=\mathrm{R}^{n} \backslash N$. We also note that condition (LR) implicitly imposes regularity properties on $V_{\ell}$. It should be noted that all derivatives in this paper are in the sense of distributions.

The Dollard-type modified free evolution is given by

$$
U_{D}(t)=U_{0}(t) \exp (i S(t,-i \nabla)) .
$$

2.2. An Existence Result. The main existence result here is stated as:

Theorem 2.3. Let $V$ satisfy Assumption 2.2. Then the wave operators

$$
W_{ \pm}^{D}=\operatorname{s-lim}_{t \rightarrow \pm \infty} U(0, t) U_{D}(t)
$$

exist.

Proof. It suffices to prove existence on a dense subset of $L^{2}\left(\mathbf{R}^{n}\right)$. Here we choose $F^{-1}\left(\mathrm{C}_{0}^{\infty}\left(\mathbf{R}^{n} \backslash N\right)\right)$. The proof is then based on the Cook method. We consider only $W_{+}^{D}$.

Let $\varphi \in F^{-1}\left(\mathfrak{C}_{0}^{\infty}\left(\mathbf{R}^{n} \backslash N\right)\right)$ and let $K=\operatorname{supp} \varphi$. We start the proof by some simple computations. We often omit the variable $t$ in the operators $M(t)$ and $D(t)$.

$$
\begin{aligned}
&\left(i \partial_{t}+\frac{1}{2} \Delta\right)(M D F M)=\left(i \partial_{t}+\frac{1}{2} \Delta\right) U_{0}=U_{0} i \partial_{t} \\
&=(M D F M) i \partial_{t}=M D F\left(i \partial_{t}-\frac{x^{2}}{2 t^{2}}\right) M \\
&=M D\left(i \partial_{t}+\frac{1}{2 t^{2}} \Delta\right) F M . \\
&\left(i \partial_{t}+\frac{1}{2} \Delta\right) M D=M D\left(i \partial_{t}+\frac{1}{2 t^{2}} \Delta\right) .
\end{aligned}
$$




$$
\begin{aligned}
\left(i \partial_{t}-H(t)\right)( & M D \exp (i S(t))) \hat{\varphi} \\
= & M D\left(i \partial_{t}+\frac{1}{2 t^{2}} \Delta-V(t, t x)\right) \exp (i S(t)) \hat{\varphi} \\
= & M D \exp (i S(t))\left\{\left(-V(t, t x)-\partial_{t} S(t)\right.\right. \\
& \left.-\frac{1}{2 t^{2}}\left((\nabla S(t))^{2}+\frac{i}{2 t^{2}} \Delta S(t)\right)\right) \hat{\varphi} \\
& \left.+\frac{1}{2 t^{2}} \Delta \hat{\varphi}+\frac{i}{t^{2}} \nabla S(t) \cdot \nabla \hat{\varphi}\right\} .
\end{aligned}
$$

Note

$$
V(t, t x)+\partial_{t} S(t)=V_{s}(t, t x)
$$

We start the Cook argument by the following computation. Assume $t>s>t_{K}$ and use (2.12) and (2.13) to get

$$
\begin{aligned}
\| U(0, t) M(t) D(t) & \exp (i S(t)) \hat{\varphi}-U(0, s) M(s) D(s) \exp (i S(s)) \hat{\varphi} \|_{2} \\
= & \left\|\int_{s}^{t} U(0, \tau)\left(i \partial_{\tau}-H(\tau)\right) M(\tau) D(\tau) \exp (i S(\tau)) \hat{\varphi} d \tau\right\|_{2} \\
\leq & \int_{s}^{t}\left\|V_{s}(\tau, \tau(\cdot)) \hat{\varphi}\right\|_{2} d \tau \\
& +\int_{s}^{t} \tau^{-2}\left(\left\|(\nabla S(\tau))^{2} \hat{\varphi}\right\|_{2}+\|(\Delta S(\tau)) \hat{\varphi}\|_{2}\right) d \tau \\
& +\int_{s}^{t} \tau^{-2}\|\nabla S(\tau) \cdot \nabla \hat{\varphi}\|_{2} d \tau \\
& +\int_{s}^{t} \tau^{-2}\|\Delta \hat{\varphi}\|_{2} d \tau .
\end{aligned}
$$

Assumption 2.2 (SR) implies that the term (2.14) tends to zero as $s, t \rightarrow \infty$. By (2.7) and (2.8) the same holds for the term (2.15). The term (2.16) is rewritten

$$
\int_{s}^{t} \tau^{-2}\|\nabla S(\tau) \cdot \nabla \hat{\varphi}\|_{2} d \tau \leq c\left(\sup _{\tau \geq t_{K}}|\tau|^{-1 / 2}\|\nabla S(\tau)\|_{L^{\infty}(K)}\right)\|\nabla \hat{\varphi}\|_{2} \int_{s}^{t} \tau^{-3 / 2} d \tau
$$

such that this term tends to zero as $s, t \rightarrow \infty$. The same fact holds trivially for the term (2.17). We conclude the existence of the limit

$$
\varphi_{+}=\lim _{t \rightarrow \infty} U(0, t) M(t) D(t) \exp (i S(t)) \hat{\varphi}
$$

in $L^{2}\left(\mathbf{R}^{n}\right)$. Now

$$
\begin{aligned}
M(t) D(t) \exp (i S(t)) \hat{\varphi} & =M(t) D(t) F M(t) M(-t) F^{-1} \exp (i S(t)) \hat{\varphi} \\
& =U_{0}(t) M(-t) \exp (i S(t,-i \nabla)) \varphi
\end{aligned}
$$


Thus we obtain

$$
\begin{aligned}
\| U_{D}(t) \varphi & -M(t) D(t) \exp (i S(t)) \hat{\varphi} \|_{2} \\
& =\|(1-M(-t)) \exp (i S(t,-i \nabla)) \varphi\|_{2} \\
& \leq t^{-1 / 2}\|\nabla(\exp (i S(t)) \hat{\varphi})\|_{2} \\
& \leq t^{-1 / 2}\|\nabla S(t)\|_{L^{\infty}(K)}\|\hat{\varphi}\|_{2}+t^{-1 / 2}\|\nabla \hat{\varphi}\|_{2}
\end{aligned}
$$

where we used $\left|1-\exp \left(i x^{2} / 2 t\right)\right| \leq|t|^{-1 / 2}|x|$ and changed to the momentum space representation. Both terms above tend to zero as $t \rightarrow \infty$, due to (2.6).

Finally we have

$$
\begin{aligned}
\left\|U(0, t) U_{D}(t) \varphi-\varphi_{+}\right\|_{2} \leq & \left\|U(0, t)\left(U_{D}(t) \varphi-M(t) D(t) \exp (i S(t)) \hat{\varphi}\right)\right\|_{2} \\
& +\left\|U(0, t) M(t) D(t) \exp (i S(t)) \hat{\varphi}-\varphi_{+}\right\|_{2} .
\end{aligned}
$$

Both terms tend to zero by the above computations.

2.3. Short Range Potentials. We give several sufficient conditions on $V_{s}(t, x)$ which imply the short range conditions in Assumption 2.2. The first result gives a condition which reduces to Enss' short range condition for a time-independent potential.

Proposition 2.4. Let $V_{s}$ satisfy

$$
\int_{|t| \geq 1}\left\|\chi\{x|| x|\geq| t \mid\} V_{s}(t, \cdot)(1-\Delta)^{-1}\right\|_{\mathcal{B}\left(L^{2}\left(\mathbf{R}^{n}\right)\right)} d t<\infty .
$$

Then Assumption 2.2 (SR) holds with $N=\{0\}$ and $m=1$.

Proof. Since $(1-\Delta) D(t)=D(t)\left(1-t^{-2} \Delta\right)$, we have

$$
D(t)(1-\Delta)^{-1}=(1-\Delta)^{-1} D(t)\left(1-t^{-2} \Delta\right)(1-\Delta)^{-1} \text {. }
$$

Take $K_{j}=\left\{x\left|j^{-1} \leq\right| x \mid \leq j\right\}, j=1,2, \ldots$ Let $\varphi \in L^{2}\left(\mathbf{R}^{n}\right)$. Then (we consider only $t>0$ )

$$
\begin{aligned}
\| \chi_{K_{j}} V_{s}(t, t(\cdot))(1 & -\Delta)^{-1} \varphi \|_{2} \\
& =\left\|\chi_{K_{j}} D(t)^{-1} V_{s}(t) D(t)(1-\Delta)^{-1} \varphi\right\|_{2} \\
& =\left\|\chi_{\left\{x\left|j^{-1} t \leq\right| x \mid \leq j t\right\}} V_{s}(t) D(t)(1-\Delta)^{-1} \varphi\right\|_{2} \\
& \leq\left\|\chi_{\left\{x\left|j^{-1} t \leq\right| x \mid\right\}} V_{s}(t)(1-\Delta)^{-1}\right\|_{\mathcal{B}\left(L^{2}\left(\mathbf{R}^{n}\right)\right)}\left(1+t^{-2}\right)\|\varphi\|_{2} .
\end{aligned}
$$

Thus (2.18) implies Assumption 2.2 (SR) with $N=\{0\}$ and $m=1$.

Let us also show that the condition used by Kuroda [12] implies our short range assumption. 
Proposition 2.5. Let $V: \mathbf{R}^{n} \rightarrow \mathbf{R}$ be a measurable function such that for some $\varepsilon>0$

$$
(1+|x|)^{-\frac{n}{2}+1+e} V \in L^{2}\left(\mathbf{R}^{n}\right) .
$$

Then $V=V_{s}$ satisfies Assumption $2.2(\mathrm{SR})$ with $N=\{0\}$ and $m>n / 4$.

Proof. Let $\varphi \in L^{2}\left(\mathbf{R}^{n}\right)$ and $K_{j}=\left\{x\left|j^{-1} \leq\right| x \mid \leq j\right\}, j=1,2, \ldots$. Then for $t>0$

$$
\begin{aligned}
\left\|\chi_{K_{j}} V(t(\cdot))(1-\Delta)^{-m} \varphi\right\|_{2} \\
\quad \leq\left\|\chi_{\left\{x\left|j^{-1} t \leq x\right| \leq j t\right\}}(1+|x|)^{-\frac{n}{2}+1+\varepsilon} V \cdot(1+|x|)^{\frac{n}{2}-1-\varepsilon} D(t)(1-\Delta)^{-m} \varphi\right\|_{2} \\
\quad \leq\left\|(1+|x|)^{-\frac{n}{2}+1+\varepsilon} V\right\|_{2} \cdot\left\|\chi_{\left\{x\left|j^{-1} t \leq\right| x \mid \leq j t\right\}}(1+|x|)^{\frac{n}{2}-1-\varepsilon} D(t)(1-\Delta)^{-m} \varphi\right\|_{\infty} \\
\quad \leq c(1+j t)^{n / 2}\left(1+j^{-1} t\right)^{-1-\varepsilon} t^{-n / 2}\left\|(1-\Delta)^{-m} \varphi\right\|_{\infty} .
\end{aligned}
$$

Since $(1-\Delta)^{-m}$ is bounded from $L^{2}\left(\mathbf{R}^{n}\right)$ to $L^{\infty}\left(\mathbf{R}^{n}\right)$ for $m>n / 4$, the result follows.

Remark 2.6. The condition (2.19) is implied by the condition $V=V_{2}+V_{p}, V_{2} \in$ $L^{2}\left(\mathbf{R}^{n}\right), V_{p} \in L^{p}\left(\mathbf{R}^{n}\right), 2<p<n$, since Hölder's inequality implies $(1+|x|)^{-\frac{n}{2}+1+\varepsilon} V_{p} \in$ $L^{2}\left(\mathbf{R}^{n}\right)$, if $\varepsilon$ is chosen sufficiently small. This condition is the one used originally by Cook, Hack, and Brownell, see the discussion in the notes of [15].

2.4. Long Range Potentials. In this section sufficient conditions for the long range condition in Assumption 2.2 are given.

Proposition 2.7. Let $V: \mathbf{R} \times \mathbf{R}^{n} \rightarrow \mathbf{R}$ be a measurable function. Assume there exists $\sigma \in L^{1}(\mathbf{R}) \cap \mathcal{C}(\mathbf{R})$ such that for any compact $K \subset \mathbf{R}^{n} \backslash N$ there exists $C_{K}>0$ such that

$$
t^{2}\|(\nabla V)(t, t(\cdot))\|_{L^{\infty}(K)}^{2}+|t|\|(\Delta V)(t, t(\cdot))\|_{L^{\infty}(K)} \leq C_{K} \sigma(t)
$$

for $t \in \mathbf{R}$. Then $V_{\ell}=V$ satisfies Assumption $2.2(\mathrm{LR})$.

Proof. Fix a compact $K \subset \mathbf{R}^{n} \backslash N$. By (2.4) and (2.20) we get

$$
\|\nabla S(t)\|_{L^{\infty}(K)} \leq C_{K}^{1 / 2} \int_{0}^{t} \sigma(\tau)^{1 / 2} d \tau
$$

and

$$
\|\Delta S(t)\|_{L^{\infty}(K)} \leq C_{K} \int_{0}^{t} \tau \sigma(\tau) d \tau .
$$

Let $1<T<t$. Then

$$
\|\nabla S(t)\|_{L^{\infty}(K)} \leq c T\left(\sup _{\tau \in[0, T]} \sigma(\tau)\right)^{1 / 2}+c(t-T)^{1 / 2} \int_{T}^{t} \sigma(\tau) d \tau
$$


and thus

$$
\limsup _{t \rightarrow \infty} t^{-1 / 2}\|\nabla S(t)\|_{L^{\infty}(K)} \leq c \int_{T}^{\infty} \sigma(\tau) d \tau .
$$

Taking $T \rightarrow \infty$ we conclude that (2.6) holds. To verify (2.7) we compute:

$$
\begin{aligned}
c \cdot \int_{1}^{T} t^{-2} \| & \nabla S(t) \|_{L^{\infty}(K)}^{2} d t \\
\leq & \int_{1}^{T} t^{-2}\left(\int_{0}^{t} \sigma(\tau)^{1 / 2} d \tau\right)^{2} d t \\
= & -T^{-1}\left(\int_{0}^{T} \sigma(\tau)^{1 / 2} d \tau\right)^{2}+\left(\int_{0}^{1} \sigma(\tau)^{1 / 2} d \tau\right)^{2} \\
& +2 \int_{1}^{T} t^{-1} \sigma(t)^{1 / 2}\left(\int_{0}^{t} \sigma(\tau)^{1 / 2} d \tau\right) d t \\
\leq & \left(\int_{0}^{1} \sigma(\tau)^{1 / 2} d \tau\right)^{2} \\
& +2\left(\int_{1}^{T} \sigma(t) d t\right)^{1 / 2} \cdot\left(\int_{1}^{T} t^{-2}\left(\int_{0}^{t} \sigma(\tau)^{1 / 2} d \tau\right)^{2} d t\right)^{1 / 2}
\end{aligned}
$$

Thus

$$
\int_{1}^{T} t^{-2}\left(\int_{0}^{t} \sigma(\tau)^{1 / 2} d \tau\right)^{2} d t \leq c+c \int_{1}^{\infty} \sigma(\tau) d \tau
$$

and we conclude that (2.7) holds. The term in (2.8) is estimated as follows:

$$
\begin{aligned}
c \cdot \int_{1}^{T} t^{-2} \| & \Delta S(t) \|_{L^{\infty}(K)} d t \\
& \leq \int_{1}^{T} t^{-2}\left(\int_{0}^{t} \tau \sigma(\tau) d \tau\right) d t \\
& =-T^{-1} \int_{0}^{T} \tau \sigma(\tau) d \tau+\int_{0}^{1} \tau \sigma(\tau) d \tau+\int_{1}^{T} \sigma(t) d t \\
& \leq c \int_{0}^{1} \tau \sigma(\tau) d \tau+\int_{1}^{\infty} \sigma(t) d t<\infty .
\end{aligned}
$$

Thus Assumption 2.2 (LR) holds for $V=V_{\ell}$.

We give three examples to illustrate the conditions in Proposition 2.7.

Example 2.8. Assume for all compact $K \subset \mathbf{R}^{n} \backslash N$

$$
\begin{aligned}
& |(\nabla V)(t, t x)| \leq c_{K}(1+|t|)^{-1-\mu / 2}, \quad x \in K, \quad \mu>1, \\
& |(\Delta V)(t, t x)| \leq c_{K}(1+|t|)^{-1-\mu^{\prime}}, \quad x \in K, \quad \mu^{\prime}>1 .
\end{aligned}
$$


Take $\sigma(t)=(1+|t|)^{-\min \left\{\mu, \mu^{\prime}\right\}}$. Then $(2.20)$ holds. As a particular case one can take

$$
V(t, x)=\lambda|x-t v|^{-\mu / 2}
$$

$\lambda \neq 0, \mu>1, v \in \mathbf{R}^{n}$, and $N=\{v\}$.

Example 2.9. Assume

$$
\begin{aligned}
& |(\nabla V)(t, t x)| \leq c_{K}(1+|t|)^{-3 / 2}(1+\log (1+|t|))^{-\mu / 2}, \quad x \in K, \quad \mu>1 \\
& |(\Delta V)(t, t x)| \leq c_{K}(1+|t|)^{-2}(1+\log (1+|t|))^{-\mu^{\prime}}, \quad x \in K, \quad \mu^{\prime}>1
\end{aligned}
$$

Take $\sigma(t)=(1+|t|)^{-1}(1+\log (1+|t|))^{-\min \left\{\mu, \mu^{\prime}\right\}}$. Then $(2.20)$ holds.

The last example is for the surfboard equation, see Section 4 . Note that in this case the definition of the wave operator is changed, replacing $U(0, t)$ by $U(1, t)$.

Example 2.10. Let $V(t, x)=\theta(t) \Phi\left(t^{-1} x\right)$. Sufficient conditions for (2.20) are $\nabla \Phi$, $\Delta \Phi \in L_{\mathrm{loc}}^{\infty}\left(\mathbf{R}^{n}\right)$, and

$$
\begin{aligned}
& \lim _{|t| \rightarrow \infty}|t|^{-1 / 2}\left|\int_{0}^{t} \theta(\tau) d \tau\right|=0 \\
& \int_{|t| \geq 1} t^{-2}\left|\int_{0}^{t} \theta(\tau) d \tau\right|^{2} d t<\infty .
\end{aligned}
$$

2.5. Stark Hamiltonians. Let $E \in \mathbf{R}^{n} \backslash\{0\}$ be fixed in the sequel. The free Stark Hamiltonian is given by $K_{0}=-\frac{1}{2} \Delta+E \cdot x$, and the full Hamiltonian with a potential $\Phi$ by $K=K_{0}+\Phi$. The wave operators for the pair $\left(K, K_{0}\right)$ exist if and only if the wave operators for $\left(H(t), H_{0}\right)$ exist, where $H(t)=H_{0}+V(t, x)$ with $V(t, x)=\Phi\left(x-\frac{1}{2} t^{2} E\right)$. This result is obtained by using the moving frame picture, see for example [9]. In this formulation the Stark Hamiltonians are covered by the present formalism. Throughout this section we use the definition

$$
V(t, x)=\Phi\left(x-\frac{1}{2} t^{2} E\right)
$$

without further comments. The coordinate along the fixed constant electric field is denoted by $x_{\|}$and the orthogonal component by $x_{\perp}$. According to this convention we also write $E=\left(E_{\|}, 0\right)$ with $E_{\|}>0$. We also decompose the gradient as $\nabla=$ $\left(\nabla_{\|}, \nabla_{\perp}\right)$. In the one-dimensional case conditions relating to $x_{\perp}$ and $\nabla_{\perp}$ are omitted.

Proposition 2.11. Let $\Phi$ satisfy

$$
\int_{1}^{\infty}\left\|\chi_{\left\{x \mid x_{\|}<-r^{2}\right\}} \Phi(x)(1-\Delta)^{-1}\right\|_{\mathcal{B}\left(L^{2}\left(\mathbf{R}^{n}\right)\right)} d r<\infty .
$$

Then $V=V_{s}$ (see (2.21)) satisfies Assumption 2.2 (SR) with $N=\emptyset, m=1$. 
Proof. Let $K_{R}=\{x|| x \mid \leq R\}$ and $t>4 R / E_{\|}$. Then

$$
\left\{t x-\frac{1}{2} t^{2} E \mid x \in K_{R}\right\} \subseteq\left\{y \mid y_{\|} \leq-\frac{1}{4} t^{2} E_{\|}\right\} .
$$

Let $\psi=(1-\Delta)^{-1} \varphi, \varphi \in L^{2}\left(\mathbf{R}^{n}\right)$. A change of variables yields

$$
\begin{aligned}
& \left\|\chi_{K_{R}} V(t, t(\cdot))(1-\Delta)^{-1} \varphi\right\|_{2} \leq\left(\int_{\left\{y \mid y_{\|} \leq-\frac{1}{4} t^{2} E_{\|\}}\right.}\left|\Phi(y) \psi\left(t^{-1} y+\frac{1}{2} t E\right)\right|^{2} d y\right)^{1 / 2} t^{-n / 2} \\
& \leq\left\|\chi_{\left\{y \mid y_{\|} \leq-\frac{1}{4} t^{2} E_{\|}\right\}} \Phi(1-\Delta)^{-1}\right\|_{\mathcal{B}\left(L^{2}\left(\mathbf{R}^{n}\right)\right)} \\
& \cdot\left(\|\psi\|_{2}+t^{-2}\|\Delta \psi\|_{2}\right)
\end{aligned}
$$

such that Assumption 2.2 (SR) follows from (2.22).

Next we look at long range potentials when the modified wave operator is the one introduced by Graf [5].

Theorem 2.12. Let $\Phi: \mathbf{R}^{n} \rightarrow \mathbf{R}$ satisfy

$$
\int_{1}^{\infty}\left\|\chi_{\left\{x \mid x_{\|}<-r^{2}\right\}}(\nabla \Phi)(1-\Delta)^{-m}\right\|_{\mathcal{B}\left(L^{2}\left(\mathbf{R}^{n}\right)\right)} r d r<\infty
$$

for some $m, 0 \leq m<\frac{1}{2}$. Let $V_{\ell}=\Phi\left(-\frac{1}{2} t^{2} E\right)$ and $V_{s}(t, x)=\Phi\left(x-\frac{1}{2} t^{2} E\right)-\Phi\left(-\frac{1}{2} t^{2} E\right)$. Then $V$ satisfies Assumption 2.2 (SR, LR).

Proof. Since $V_{\ell}$ is independent of $x$, the (LR)-conditions are trivially satisfied. We write

$$
V_{s}(t, t x)=\int_{0}^{1}(t x \cdot \nabla \Phi)\left(t \sigma x-\frac{1}{2} t^{2} E\right) d \sigma .
$$

Fix $R>0$ and $t>4 R / E_{\|}$. Using the change of variables as in the previous proof we get with $\psi=(1-\Delta)^{-m} \varphi, \varphi \in L^{2}\left(\mathrm{R}^{n}\right)$,

$$
\begin{aligned}
\left\|\chi_{K_{R}} V_{s}(t, t(\cdot)) \psi\right\|_{2} \leq & R t\left\|\chi_{\left\{y \mid y_{\|} \leq-\frac{1}{4} t^{2} E_{\|}\right\}}(\nabla \Phi)(1-\Delta)^{-m}\right\|_{\mathcal{B}\left(L^{2}\left(\mathbf{R}^{n}\right)\right)} \\
& \cdot \int_{0}^{1}(\sigma t)^{-n / 2}\left\|(1-\Delta)^{m}\left(\psi\left((t \sigma)^{-1}(\cdot)\right)\right)\right\|_{2} d \sigma \\
\leq & c R t\left\|\chi_{\left\{y \mid y_{\|} \leq-\frac{1}{4} t^{2} E_{\|}\right\}}(\nabla \Phi)(1-\Delta)^{-m}\right\|_{\mathcal{B}\left(L^{2}\left(\mathbf{R}^{n}\right)\right)} \\
& \cdot \int_{0}^{1}\left(1+(t \sigma)^{-2 m}\right) d \sigma\|\varphi\|_{2} .
\end{aligned}
$$

Since $2 m<1$, condition (2.23) shows that $V_{s}$ satisfies Assumption $2.2(\mathrm{SR})$.

Let us note that Graf's conditions $\Phi \in \mathcal{C}^{1}\left(\mathrm{R}^{n}\right), \nabla \Phi(y)=O\left(|y|^{-1-\varepsilon}\right)$ as $|y| \rightarrow \infty$ imply (2.23). Our conditions allow some local singularities in $\nabla \Phi$. If we impose further conditions on $\Phi$, then we can allow slower decay in the direction opposite the electric field. 
Theorem 2.13. Let $\Phi \in \mathcal{C}^{2}\left(\mathbf{R}^{n}\right)$. Assume for some $m, 0 \leq m<\frac{1}{2}$

$$
\begin{aligned}
& \lim _{r \rightarrow \infty} \Phi(-r E)=0, \\
& \int_{1}^{\infty}\left\|\chi_{\left\{x \mid x_{\|}<-r^{2}\right\}}\left(\nabla_{\perp} \Phi\right)(1-\Delta)^{-m}\right\|_{\mathcal{B}\left(L^{2}\left(\mathbf{R}^{n}\right)\right)} r d r<\infty, \\
& \int_{1}^{\infty}\left\|\chi_{\left\{x \mid x_{\|}<-r^{2}\right\}}\left(\nabla_{\|}(\nabla \Phi)\right)(1-\Delta)^{-m}\right\|_{\mathcal{B}\left(L^{2}\left(\mathbf{R}^{n}\right)\right)} r^{2} d r<\infty .
\end{aligned}
$$

Then the modified wave operators $W_{ \pm}^{D}$ exist with the choice $V_{\ell}(t, x)=\Phi\left(-\frac{1}{2} t^{2} E\right)$.

Proof. Assumption 2.1 is satisfied, since $V$ is bounded and continuous, see e.g. [23]. We take

$$
S(t, y)=-\int_{0}^{t} \Phi\left(-\frac{1}{2} \tau^{2} E\right) d \tau+y_{\|} \Phi\left(-\frac{1}{2} t^{2} E\right)
$$

We have

$$
\left\|\left\{\exp \left(-i \int_{0}^{t} \Phi\left(-\frac{1}{2} \tau^{2} E\right) d \tau\right)-\exp (i S(t,-i \nabla))\right\} \varphi\right\|_{2}=\left\|\left(1-\exp \left(i \xi_{\|} \Phi\left(-\frac{1}{2} t^{2} E\right)\right)\right) \hat{\varphi}\right\|_{2}
$$

which tends to zero as $t \rightarrow \infty$ by (2.24) and dominated convergence. Thus we can use this $S(t, y)$ to prove existence of the Graf modified wave operators. We calculate:

$$
\begin{aligned}
V_{s}(t, t x)= & \Phi\left(t x-\frac{1}{2} t^{2} E\right)+\partial_{t} S(t, x) \\
= & \Phi\left(t x-\frac{1}{2} t^{2} E\right)-\Phi\left(-\frac{1}{2} t^{2} E\right)-t x_{\|}\left(\nabla_{\|} \Phi\right)\left(-\frac{1}{2} t^{2} E\right) \\
= & \int_{0}^{1}(t x \cdot \nabla \Phi)\left(t \sigma x-\frac{1}{2} t^{2} E\right) d \sigma-t x_{\|}\left(\nabla_{\|} \Phi\right)\left(-\frac{1}{2} t^{2} E\right) \\
= & \int_{0}^{1}\left(t x_{\perp} \cdot \nabla_{\perp} \Phi\right)\left(t \sigma x-\frac{1}{2} t^{2} E\right) d \sigma \\
& +\int_{0}^{1}\left\{\left(\nabla_{\|} \Phi\right)\left(t \sigma x-\frac{1}{2} t^{2} E\right)-\left(\nabla_{\|} \Phi\right)\left(-\frac{1}{2} t^{2} E\right)\right\} d \sigma t x_{\|} \\
= & \int_{0}^{1}\left(t x_{\perp} \cdot \nabla_{\perp} \Phi\right)\left(t \sigma x-\frac{1}{2} t^{2} E\right) d \sigma \\
& +t x_{\|} \int_{0}^{1} \int_{0}^{1}\left(t \sigma x \cdot \nabla\left(\nabla_{\|} \Phi\right)\right)\left(t \sigma \theta x-\frac{1}{2} t^{2} E\right) d \theta d \sigma .
\end{aligned}
$$

Repeating the estimates in the proof of Theorem 2.12 and using (2.25), (2.26), we get integrability of $\left\|\chi_{K_{R}} V_{s}(t, t(\cdot))(1-\Delta)^{-m} \varphi\right\|_{2}$, with $0 \leq m<\frac{1}{2}$. 
The argument in the proof of Theorem 2.13 can be refined further. To do this we take

$$
S(t, y)=-\int_{0}^{t} \Phi\left(-\frac{1}{2} \tau^{2} E\right) d \tau+y_{\|} \sum_{j=0}^{N} \frac{\left(t y_{\|}\right)^{j}}{(j+1) !}\left(\nabla_{\|}^{j} \Phi\right)\left(-\frac{1}{2} t^{2} E\right)
$$

Then the proof of the following result is straightforward. Further details are omitted.

Proposition 2.14. Assume $\Phi \in \mathfrak{C}^{N+2}\left(\mathrm{R}^{n}\right)$ for some integer $N \geq 0$. Assume for some $m, 0 \leq m<\frac{1}{2}$,

$$
\begin{gathered}
\lim _{r \rightarrow \infty} r^{j / 2} \nabla_{\|}^{j} \Phi(-r E)=0, \quad j=0,1,2 \ldots, N, \\
\int_{1}^{\infty}\left\|\chi_{\left\{x \mid x_{\|}<-r^{2}\right\}}\left(\nabla_{\|}^{j} \Phi\right)(1-\Delta)^{-m}\right\|_{\mathcal{B}\left(L^{2}\left(\mathbf{R}^{n}\right)\right)^{r}} r^{j-1} d r<\infty, \quad j=1,2 \ldots, N \\
\int_{1}^{\infty}\left\|\chi_{\left\{x \mid x_{\|}<-r^{2}\right\}}\left(\nabla_{\|}^{j} \nabla_{\perp} \Phi\right)(1-\Delta)^{-m}\right\|_{\mathcal{B}\left(L^{2}\left(\mathbf{R}^{n}\right)\right)^{r}} r^{j+1} d r<\infty, \quad j=1,2 \ldots, N, \\
\int_{1}^{\infty}\left\|\chi_{\left\{x \mid x_{\|}<-r^{2}\right\}}\left(\nabla_{\|}^{N+1}(\nabla \Phi)\right)(1-\Delta)^{-m}\right\|_{\mathcal{B}\left(L^{2}\left(\mathbf{R}^{n}\right)\right)} r^{N+2} d r<\infty .
\end{gathered}
$$

Then the modified wave operators $W_{ \pm}^{D}$ exist with the choice $V_{\ell}(t, x)=\Phi\left(-\frac{1}{2} t^{2} E\right)$.

In the one-dimensional case we can obtain the following result based on the modified wave operator introduced in [9]. In this case we take $E=1$ to simplify the notation.

Proposition 2.15. Assume $\Phi \in L^{\infty}(\mathbf{R}) \cap \mathcal{C}^{2}(\mathbf{R})$ with

$$
\int_{1}^{\infty}\left\|\chi_{\left\{x \mid x<-r^{2}\right\}} \Phi^{\prime}\right\|_{\infty} r^{-1} d r<\infty .
$$

Let $V(t, x)=V_{\ell}(t, x)=\Phi\left(x-\frac{1}{2} t^{2}\right)$. Then $V$ satisfies Assumption $2.2(\mathrm{LR})$.

Proof. Let $K_{R}=\{x|| x \mid \leq R\}$ and assume $t>4(R+1)$. Following the computations in [9] we find for $x \in K_{R}$

$$
\int_{0}^{t} \tau \Phi^{\prime}\left(\tau x-\frac{1}{2} \tau^{2}\right) d \tau=\left(\int_{0}^{R+1}+\int_{R+1}^{t}\right) \tau \Phi^{\prime}\left(\tau x-\frac{1}{2} \tau^{2}\right) d \tau
$$

We now estimate:

$$
\left|\int_{0}^{R+1} \tau \Phi^{\prime}\left(\tau x-\frac{1}{2} \tau^{2}\right) d \tau\right| \leq(R+1)^{2} \sup _{|y| \leq 2(R+1)^{2}}\left|\Phi^{\prime}(y)\right|
$$


and

$$
\begin{aligned}
\left|\int_{R+1}^{t} \tau \Phi^{\prime}\left(\tau x-\frac{1}{2} \tau^{2}\right) d \tau\right|= & \mid-t(t-x)^{-1} \Phi\left(t x-\frac{1}{2} t^{2}\right) \\
& +(R+1)(R+1-x)^{-1} \Phi\left((R+1) x-\frac{1}{2}(R+1)^{2}\right) \\
& -\int_{R+1}^{t} x(x-\tau)^{-2} \Phi\left(\tau x-\frac{1}{2} \tau^{2}\right) d \tau \mid \\
\leq & 2\|\Phi\|_{\infty}+(R+1)\|\Phi\|_{\infty}+R\|\Phi\|_{\infty} \int_{R+1}^{\infty}(\tau-R)^{-2} d \tau
\end{aligned}
$$

These computations imply

$$
\|\nabla S(t)\|_{L^{\infty}(K)} \leq c, \quad t>4(R+1),
$$

such that (2.6) and (2.7) hold. Similarly, we have

$$
\begin{aligned}
\int_{R+1}^{t} \tau^{2} \Phi^{\prime \prime}(\tau x & \left.-\frac{1}{2} \tau^{2}\right) d \tau=t^{2}(x-t)^{-1} \Phi^{\prime}\left(t x-\frac{1}{2} t^{2}\right) \\
& -(R+1)^{2}(x-R-1)^{-1} \Phi^{\prime}\left((R+1) x-\frac{1}{2}(R+1)^{2}\right) \\
& +\left\{(x-t)^{-1}-x^{2}(x-t)^{-3}\right\} \Phi\left(t x-\frac{1}{2} t^{2}\right) \\
& -\left\{(x-R-1)^{-1}-x^{2}(x-R-1)^{-3}\right\} \Phi\left((R+1) x-\frac{1}{2}(R+1)^{2}\right) \\
& -\int_{R+1}^{t}\left((x-\tau)^{-2}-3 x^{2}(x-\tau)^{-4}\right) \Phi\left(\tau x-\frac{1}{2} \tau^{2}\right) d \tau
\end{aligned}
$$

We estimate

$$
\left|\int_{R+1}^{t} \tau^{2} \Phi^{\prime \prime}\left(\tau x-\frac{1}{2} \tau^{2}\right) d \tau\right| \leq 2 t\left|\Phi^{\prime}\left(t x-\frac{1}{2} t^{2}\right)\right|+c \sup _{|y| \leq 2(R+1)^{2}}\left|\Phi^{\prime}(y)\right|+c\|\Phi\|_{\infty} .
$$

and

$$
\left|\int_{0}^{R+1} \tau^{2} \Phi^{\prime \prime}\left(\tau x-\frac{1}{2} \tau^{2}\right) d \tau\right| \leq(R+1)^{3} \sup _{|y| \leq 2(R+1)^{2}}\left|\Phi^{\prime \prime}(y)\right|
$$

Combining these estimates we get

$$
t^{-2}\|\Delta S(t)\|_{L^{\infty}(K)} \leq C t^{-1}\left\|\chi_{\left\{y \mid y \leq-\frac{1}{4} t^{2}\right\}} \Phi^{\prime}(y)\right\|_{\infty}+c t^{-2}
$$

which is integrable by $(2.27)$.

We now use this result to resolve the discrepancy between classical and quantum scattering discovered in [8]. We use Proposition 5.1 together with Proposition 2.15. See the discussion in Section 5. 
Theorem 2.16. Assume $\Phi \in L^{\infty}(\mathbf{R}) \cap \mathcal{C}^{2}(\mathbf{R})$ with

$$
\begin{gathered}
\lim _{r \rightarrow \infty} \Phi\left(-r^{2}\right)=0, \\
\int_{1}^{\infty}\left(\left\|\chi_{\left\{x \mid x<-r^{2}\right\}} \Phi\right\|_{\infty}+\left\|\chi_{\left\{x \mid x<-r^{2}\right\}} \Phi^{\prime}\right\|_{\infty}\right) r^{-1} d r<\infty .
\end{gathered}
$$

Let $V(t, x)=\Phi\left(x-\frac{1}{2} t^{2}\right)$. Then the modified wave operators with the Graf modifier $S_{G}(t)=-\int_{0}^{t} \Phi\left(-\frac{1}{2} \tau^{2}\right) d \tau$ exist.

Proof. We consider only the $W_{+}^{D}$-case. We introduce

$$
S(t, y)=-\int_{0}^{t} \Phi\left(\tau y-\frac{1}{2} \tau^{2}\right) d \tau
$$

We will prove that for any compact $K \subset \mathrm{R}^{n}$ the limit

$$
\lim _{t \rightarrow \infty}\left(S(t, y)-S_{G}(t)\right)
$$

exists uniformly for $y \in K$. Then combining this result with Proposition 5.1 and Proposition 2.15 the theorem follows.

Assume $K \subseteq[-R+1, R-1]$ for some $R>1$. Then for $t>R$ and $y \in K$

$$
S_{G}(t)-S(t, y)=\left(\int_{0}^{R}+\int_{R}^{t}\right)\left(\Phi\left(\tau y-\frac{1}{2} \tau^{2}\right)-\Phi\left(-\frac{1}{2} \tau^{2}\right)\right) d \tau .
$$

The integral from 0 to $R$ tends to zero by (2.28). We now integrate by parts

$$
\begin{aligned}
\int_{R}^{t}\left(\Phi\left(\tau y-\frac{1}{2} \tau^{2}\right)-\right. & \left.\Phi\left(-\frac{1}{2} \tau^{2}\right)\right) d \tau=\int_{R}^{t} \int_{0}^{1} \tau y \Phi^{\prime}\left(\tau \theta y-\frac{1}{2} \tau^{2}\right) d \theta d \tau \\
= & \int_{0}^{1}\left(\frac{t y}{\theta y-t} \Phi\left(t \theta y-\frac{1}{2} t^{2}\right)-\frac{R y}{\theta y-R} \Phi\left(R \theta y-\frac{1}{2} R^{2}\right)\right) d \theta \\
& -\int_{0}^{1} \int_{R}^{t}\left(\frac{y}{\theta y-\tau}+\frac{\tau y}{(\theta y-\tau)^{2}}\right) \Phi\left(\tau \theta y-\frac{1}{2} \tau^{2}\right) d \tau d \theta
\end{aligned}
$$

Now

$$
\frac{t y}{\theta y-t} \Phi\left(t \theta y-\frac{1}{2} t^{2}\right) \rightarrow 0 \quad \text { as } t \rightarrow \infty,
$$

uniformly for $y \in K, \theta \in[0,1]$, so the first term vanishes. Furthermore,

$$
\left|\left(\frac{y}{\theta y-\tau}+\frac{\tau y}{(\theta y-\tau)^{2}}\right) \Phi\left(\tau \theta y-\frac{1}{2} \tau^{2}\right)\right| \leq c \frac{1}{\tau}\left|\Phi\left(\tau \theta y-\frac{1}{2} \tau^{2}\right)\right|
$$

which is integrable from $R$ to $\infty$ by (2.29). This proves the result and hence the theorem. 


\section{Non-Existence of Wave Operators}

We give a series of results on non-existence of the $\operatorname{limits}_{t \rightarrow \infty} U(0, t) U_{0}(t) \varphi$ and $\lim _{t \rightarrow \infty} U(0 ; t) U_{D}(t) \varphi$. Results for the limit $t \rightarrow-\infty$ are entirely analogous and are omitted.

3.1. Non-Existence Results for Ordinary Wave Operators. We give a connection between the existence of $\lim _{t \rightarrow \infty} U(0, t) U_{0}(t) \varphi$ in $L^{2}\left(\mathbf{R}^{n}\right)$ and the supports of $\hat{\varphi}$ and a certain scaling limit of $V$. We recall that throughout the paper $N \subset \mathbf{R}^{n}$ is a closed set of measure zero, depending on the potential under consideration.

Assumption 3.1. Let $V$ satisfy Assumption 2.1. Assume there exist $t_{0}>0, W \in$ $L_{\text {loc }}^{2}\left(\mathbf{R}^{n} \backslash N\right), m \geq 0$, and a function $\theta \in \mathcal{C}\left(\left[t_{0}, \infty\right), \mathrm{R}_{+}\right)$with $\int_{t_{0}}^{\infty} \theta(t) d t=+\infty$. Furthermore, to each compact $K \subset \mathbf{R}^{n} \backslash N$ there exist $\sigma_{K} \in \mathcal{C}\left(\left[t_{0}, \infty\right), \mathbf{R}_{+}\right)$with $\lim _{t \rightarrow \infty} \sigma_{K}(t)=0$, and $\rho_{K} \in L^{1}\left(\left[t_{0}, \infty\right)\right), \rho_{K} \geq 0$, such that for $t \geq t_{0}$

$$
\left\|\chi_{K}\left\{D(t)^{-1} V(t, \cdot) D(t)-\theta(t) W\right\}(1-\Delta)^{-m}\right\|_{\mathcal{B}\left(L^{2}\left(\mathbf{R}^{n}\right)\right)} \leq \sigma_{K}(t) \theta(t)+\rho_{K}(t) \cdot(3.1)
$$

Theorem 3.2. Let $V$ satisfy Assumption 3.1.

(i) Let $\varphi \in L^{2}\left(\mathbf{R}^{n}\right)$. Assume $\lim _{t \rightarrow \infty} U(0, t) U_{0}(t) \varphi$ exists in $L^{2}\left(\mathbf{R}^{n}\right)$. Then $W \hat{\varphi}=$ 0 .

(ii) Let $\varphi \in L^{2}\left(\mathrm{R}^{n}\right)$. Assume $\sigma_{K} \theta \in L^{1}\left(\left[t_{0}, \infty\right)\right)$ for any compact $K \subset \mathbf{R}^{n} \backslash N$. Assume $W \hat{\varphi}=0$. Then $\lim _{t \rightarrow \infty} U(0, t) U_{0}(t) \varphi$ exists in $L^{2}\left(\mathbf{R}^{n}\right)$.

Proof. To prove the first part we take $\psi \in \mathcal{S}\left(\mathbf{R}^{n}\right)$, the Schwartz space, such that $\hat{\psi} \in \mathcal{C}_{0}^{\infty}\left(\mathbf{R}^{n} \backslash N\right)$ and assume $K \supseteq \operatorname{supp} \hat{\psi}$. Let $\varphi_{+}=\lim _{t \rightarrow \infty} U(0, t) U_{0}(t) \varphi$. We assume $(\hat{\psi}, W \hat{\varphi}) \neq 0$ and show that it leads to a contradiction. Let $t>s>t_{0}$. We compute:

$$
\begin{aligned}
i(U(0, t) & \left.M(t) D(t) \hat{\psi}-U(0, s) M(s) D(s) \hat{\psi}, \varphi_{+}\right) \\
= & \int_{s}^{t}\left(U(0, \tau)\left(i \partial_{\tau}-H(\tau)\right) M(\tau) D(\tau) \hat{\psi}, \varphi_{+}\right) d \tau \\
= & -\int_{s}^{t}\left(M D\left(D^{-1} V(\tau) D \hat{\psi}\right), M D \hat{\varphi}\right) d \tau \\
& +\int_{s}^{t}\left(M D\left(D^{-1} V(\tau) D \hat{\psi}\right), M D F(\varphi-M \varphi)\right) d \tau \\
& +\int_{s}^{t}\left(M D\left(D^{-1} V(\tau) D \hat{\psi}\right), U_{0}(\tau) \varphi-U(\tau, 0) \varphi_{+}\right) d \tau \\
& +\int_{s}^{t}\left(M D \frac{1}{2 \tau^{2}} \Delta \hat{\psi}, U(\tau, 0) \varphi_{+}\right) d \tau
\end{aligned}
$$


These four terms are estimated as follows. The first term (3.2) is estimated by

$$
\begin{aligned}
\left|\int_{s}^{t}\left(D^{-1} V(\tau) D \hat{\psi}, \hat{\varphi}\right) d \tau\right|= & \mid \int_{s}^{t} \theta(\tau)(W \hat{\psi}, \hat{\varphi}) d \tau \\
& +\int_{s}^{t}\left(\chi_{K}\left(D^{-1} V(\tau) D-\theta(\tau) W\right) \hat{\psi}, \hat{\varphi}\right) d \tau \mid \\
\geq & |(W \hat{\psi}, \hat{\varphi})| \int_{s}^{t} \theta(\tau) d \tau \\
& -\int_{s}^{t}\left(\sigma_{K}(\tau) \theta(\tau)+\rho_{K}(\tau)\right) d \tau \cdot\left\|(1-\Delta)^{m} \hat{\psi}\right\|_{2}\|\hat{\varphi}\|_{2} .
\end{aligned}
$$

The second term (3.3) is estimated by

$$
\begin{aligned}
\left|\int_{s}^{t}(V(\tau, \tau(\cdot)) \hat{\psi}, F(\varphi-M \varphi)) d \tau\right| \leq \int_{s}^{t}\left\|\chi_{K} V(\tau, \tau(\cdot)) \hat{\psi}\right\|_{2}\|(1-M(\tau)) \varphi\|_{2} d \tau \\
\leq \int_{s}^{t}\left\{\left\|\chi_{K}\left\{D(\tau)^{-1} V(\tau) D(\tau)-\theta(\tau) W\right\}(1-\Delta)^{-m}\right\|_{\mathcal{B}\left(L^{2}\left(\mathbf{R}^{n}\right)\right)}\right. \\
\left.\cdot\left\|(1-\Delta)^{m} \hat{\psi}\right\|_{2}+\theta(\tau)\|W \hat{\psi}\|_{2}\right\}\|(1-M(\tau)) \varphi\|_{2} d \tau \\
\leq \int_{s}^{t}\left\{\left(\sigma_{K}(\tau) \theta(\tau)+\rho_{K}(\tau)\right)\left\|(1-\Delta)^{m} \hat{\psi}\right\|_{2}+\theta(\tau)\|W \hat{\psi}\|_{2}\right\} \\
\cdot\|(1-M(\tau)) \varphi\|_{2} d \tau .
\end{aligned}
$$

The term (3.4) is estimated in the same manner:

$$
\begin{gathered}
\int_{s}^{t}\left\|\chi_{K} V(\tau, \tau(\cdot)) \hat{\psi}\right\|_{2}\left\|U(\tau) \varphi-U(\tau, 0) \varphi_{+}\right\|_{2} d \tau \\
\leq \int_{s}^{t}\left\{\left(\sigma_{K}(\tau) \theta(\tau)+\rho_{K}(\tau)\right)\left\|(1-\Delta)^{m} \hat{\psi}\right\|_{2}+\theta(\tau)\|W \hat{\psi}\|_{2}\right\} \\
\cdot\left\|U(\tau) \varphi-U(\tau, 0) \varphi_{+}\right\|_{2} d \tau
\end{gathered}
$$

Finally (3.5) is estimated by

$$
\int_{s}^{t} \tau^{-2}\|\Delta \hat{\psi}\|_{2}\left\|\varphi_{+}\right\|_{2} d \tau
$$

Fix $\varepsilon>0$. If we note that $\lim _{t \rightarrow \infty} \sigma_{K}(t)=0$, we can determine $T(\varepsilon)>t_{0}$ such that for $t>s>T(\varepsilon)$

$$
\begin{aligned}
2\|\psi\|_{2}\left\|\varphi_{+}\right\|_{2} & \geq\left|\left(U(0, t) M(t) D(t) \hat{\psi}-U(0, s) M(s) D(s) \hat{\psi}, \varphi_{+}\right)\right| \\
& \geq\{|(\hat{\psi}, W \hat{\varphi})|-\varepsilon\} \int_{s}^{t} \theta(\tau) d \tau-c s^{-1}-C \int_{s}^{\infty} \rho_{K}(\tau) d \tau
\end{aligned}
$$


We conclude that $(\hat{\psi}, W \hat{\varphi})=0$. Since $\hat{\psi}$ varies over a dense set, we conclude $W \hat{\varphi}=0$. This proves the first part of the theorem.

To prove the second part it suffices to consider $\varphi \in \mathcal{S}\left(\mathbf{R}^{n}\right)$ with $\hat{\varphi} \in \mathcal{C}_{0}^{\infty}\left(\mathbf{R}^{n} \backslash N\right)$, $W \hat{\varphi}=0$, by a density argument. Let $K=\operatorname{supp} \hat{\varphi}$. Using $(2.11)$ and $W \hat{\varphi}=0$ we get

$$
\begin{aligned}
& \|\left(U(0, t) M(t) D(t) \hat{\varphi}-U(0, s) M(s) D(s) \hat{\varphi} \|_{2}\right. \\
&=\left\|\int_{s}^{t} U(0, \tau)\left(i \partial_{\tau}-H(\tau)\right) M(\tau) D(\tau) \hat{\varphi} d \tau\right\|_{2} \\
& \leq \int_{s}^{t}\left\|D(\tau)^{-1} V(\tau) D(\tau) \hat{\varphi}\right\|_{2} d \tau+\frac{1}{2} \int_{s}^{t} \tau^{-2}\|\Delta \hat{\varphi}\|_{2} d \tau \\
& \leq \int_{s}^{t}\left\|\chi_{K}\left\{D(\tau)^{-1} V(\tau) D(\tau)-\theta(\tau) W\right\}(1-\Delta)^{-m}\right\|_{\mathcal{B}\left(L^{2}\left(\mathbf{R}^{n}\right)\right)} \\
& \cdot\left\|(1-\Delta)^{m} \hat{\varphi}\right\|_{2} d \tau+\int_{s}^{t} \tau^{-2}\|\Delta \hat{\varphi}\|_{2} d \tau .
\end{aligned}
$$

The result now follows from Assumption 3.1.

This theorem states in a fairly precise manner that $O\left(|x|^{-1}\right)$ is the borderline for the existence of the ordinary wave operators, if one excludes oscillation in the potential. Let us illustrate this fact by a few examples.

Example 3.3. Let $V=V_{1}+V_{2}, V_{1}(t, x)=\lambda\left|x-t v_{1}\right|^{-\mu_{1}}, \lambda \neq 0, v_{1} \in \mathbf{R}^{n}, 0<\mu_{1} \leq 1$, and $\left|V_{2}(t, x)\right| \leq\left|x-t v_{2}\right|^{-\mu_{2}}, \mu_{2}>\mu_{1}$. Let $N=\left\{v_{1}, v_{2}\right\}$ and let $K \subset \mathbf{R}^{n} \backslash N$ be compact. Let $\theta(t)=t^{-\mu_{1}}$ and $W(x)=\lambda\left|x-v_{1}\right|^{-\mu_{1}}$. Then

$$
\left\|\chi_{K}\left(D(t)^{-1} V(t) D(t)-\theta(t) W\right)\right\|_{\mathcal{B}\left(L^{2}\left(\mathbf{R}^{n}\right)\right)} \leq c t^{-\mu_{2}}=\sigma_{K}(t) \theta(t)
$$

with $\sigma_{K}(t)=c t^{\mu_{1}-\mu_{2}}$. Thus the conditions in Assumption 3.1 are satisfied. (To verify Assumption 2.1 one can use results in [23].) The logarithmic borderline case can also be obtained in this type of example. Take $V=V_{1}+V_{2}$ with

$$
V_{1}(t, x)=\lambda\left|x-t v_{1}\right|^{-1}\left(1+\log \left(1+\left|x-t v_{1}\right|\right)\right)^{-1}
$$

and

$$
\left|V_{2}(t, x)\right| \leq c\left|x-t v_{2}\right|^{-1}\left(1+\log \left(1+\left|x-t v_{2}\right|\right)\right)^{-\mu}
$$

with $\mu>1$. Further details are omitted.

Example 3.4. Let $V=V_{1}+V_{2}, V_{1}(t, x)=\lambda\left|x^{2}-t^{2}\right|^{-\mu_{1} / 2}, \lambda \neq 0, v_{1} \in \mathbf{R}^{n}, 0<\mu_{1} \leq 1$, and $\left|V_{2}(t, x)\right| \leq|| x|-| t||^{-\mu_{2}}, \mu_{2}>\mu_{1}$. Then we take $N=\{x|| x \mid=1\}, \theta(t)=t^{-\mu_{1}}$, $\sigma_{K}(t)=c t^{\mu_{1}-\mu_{2}}, W(x)=\lambda\left|1-x^{2}\right|^{-\mu_{1} / 2}$. Further details are omitted.

Example 3.5. $V(t, x)=\lambda\left|x-t^{k} v\right|^{-\mu}, v \in \mathbf{R}^{n} \backslash\{0\}, k>1,0<\mu \leq 1 / k$. Take

$$
\sigma_{K}(t)=\lambda \min \left\{|| t^{1-k} x-\left.v\right|^{-\mu}-|v|^{-\mu}|| x \in K\right\},
$$

$\theta(t)=t^{-\mu k}$, and $W(x)=\lambda|v|^{-\mu}$. For $k=2$ this example yields the result in [14]. 
We also give an example for the surfboard equation, see Section 4 . Note that the definition of the wave operator is changed in this case, replacing $U(0, t)$ by $U(1, t)$.

Example 3.6. Let $V(t, x)=\theta(t) \Phi\left(t^{-1} x\right)$. Let $\Phi \in L_{\text {loc }}^{2}\left(\mathbf{R}^{n} \backslash N\right)$. We can take $\theta(t)=$ $t^{-\mu}, 0<\mu \leq 1$, or $\theta(t)=t^{-1}(\log t)^{-\mu}, 0<\mu \leq 1$, or $\theta(t)=t^{-1}\left|\sin \left(t^{\lambda}\right)\right|, \lambda>0$, $0<\mu \leq 1$. In these cases the conditions in Assumption 3.1 are satisfied.

3.2. Stark Hamiltonians. For the Stark Hamiltonian with Graf's modifier we have the following result:

Theorem 3.7. Assume $\Phi \in L^{\infty}\left(\mathbf{R}^{n}\right) \cap \mathcal{C}^{2}\left(\mathbf{R}^{n}\right), E \in \mathbf{R}^{n} \backslash\{0\}$. Let $V_{\ell}(t, x)=$ $\Phi\left(-\frac{1}{2} t^{2} E\right), V_{s}(t, x)=\Phi\left(x-\frac{1}{2} t^{2} E\right)-\Phi\left(-\frac{1}{2} t^{2} E\right)$. Assume there exists $\theta \in \mathcal{C}\left(\mathbf{R}_{+}\right)$ with $\theta \geq 0, \int_{0}^{\infty} \theta(t) d t=\infty$. Assume there exists $W \in L_{\text {loc }}^{2}\left(\mathbf{R}^{n} \backslash N\right)$ such that for all compact $K \subset \mathbf{R}^{n} \backslash N$,

$$
\lim _{t \rightarrow \infty}\left\|\chi_{K}\left(\theta(t)^{-1} D(t)^{-1} V_{s}(t) D(t)-W\right)\right\|_{\mathcal{B}\left(L^{2}\left(\mathbf{R}^{n}\right)\right)}=0 .
$$

Let $\varphi \in L^{2}\left(\mathbf{R}^{n}\right)$. Assume $\lim _{t \rightarrow \infty} U(0, t) U_{D}(t) \varphi$ exists. Then $W \hat{\varphi}=0$.

Proof. The line of proof is very close to the one for part (i) of Theorem 3.2. We note

$$
\begin{aligned}
\left(i \partial_{\tau}+\frac{1}{2} \Delta\right) M(\tau) D(\tau) \exp (i S(\tau)) \hat{\psi} \\
\quad=M(\tau) D(\tau) \exp (i S(\tau))\left(\frac{1}{2 \tau^{2}} \Delta+\Phi\left(-\frac{1}{2} \tau^{2} E\right)\right) \hat{\psi}
\end{aligned}
$$

Since the extra term is a function of $\tau$ only, we can replace $D(\tau)^{-1} V(\tau) D(\tau)$ by

$$
D(\tau)^{-1} V(\tau) D(\tau)-\Phi\left(-\frac{1}{2} \tau^{2} E\right)=D(\tau)^{-1} V_{s}(\tau) D(\tau)
$$

Now the arguments can be repeated, with $\rho_{K} \equiv 0$ and

$$
\sigma_{K}(t)=\left\|\chi_{K}\left(\theta(t)^{-1} D(t)^{-1} V_{s}(t) D(t)-W\right)\right\|_{\mathcal{B}\left(L^{2}\left(\mathbf{R}^{n}\right)\right)} \cdot
$$

Further details are omitted.

Example 3.8. Take dimension $n=2$ and let $E=(2,0)$,

$$
\Phi\left(x_{1}, x_{2}\right)=\frac{x_{2}}{\left(1+x^{2}\right)^{1 / 2}\left(1+\log \left(1+x^{2}\right)\right)} .
$$

Let $N=\{0\}, \theta(t)=(2 t \log t)^{-1}$, and $W\left(x_{1}, x_{2}\right)=x_{2}$. Then the conditions in Theorem 3.7 are satisfied, so both the usual wave operators and the Graf modified wave operators fail to exist. Note that in this example $\nabla \Phi(x)=O\left((|x| \log |x|)^{-1}\right)$ as $|x| \rightarrow \infty$. 
3.3. Non-Existence of Modified Wave Operators. We give a result on the non-existence of a Dollard type modified wave operator.

Assumption 3.9. Let $V_{l}=V$ satisfy Assumption 2.1. Assume there exist $t_{0}>0$, $W_{1} \in L_{\text {loc }}^{\infty}\left(\mathbf{R}^{n} \backslash N\right)$, and $\theta_{1} \in \mathcal{C}\left(\mathbf{R}_{+}\right)$with $\theta_{1} \geq 0, \int_{t_{0}}^{\infty} \theta_{1}(\tau) d \tau=\infty$, such that for every compact $K \subset \mathbf{R}^{n} \backslash N$ one can find $t_{K}>t_{0}, \sigma_{K} \in \mathcal{C}\left(\mathbf{R}_{+}\right), \sigma_{K} \geq 0, \lim _{t \rightarrow \infty} \sigma_{K}(t)=0$, $\rho_{K} \in L^{1}\left(\left[t_{K}, \infty\right)\right)$ with the property

$$
\left\|t^{-2}(\nabla S(t))^{2}-\theta_{1}(t) W_{1}\right\|_{L^{\infty}(K)}+t^{-2}\|\Delta S(t)\|_{L^{\infty}(K)} \leq \sigma_{K}(t) \theta_{1}(t)+\rho_{K}(t) .
$$

Assume furthermore

$$
\lim _{t \rightarrow \infty} t^{-1 / 2}\|\nabla S(t)\|_{L^{\infty}(K)}=0 .
$$

Theorem 3.10. Let $V$ satisfy Assumption 3.9. Let $\varphi \in L^{2}\left(\mathbf{R}^{n}\right)$. Assume that $\lim _{t \rightarrow \infty} U(0, t) U_{D}(t) \varphi$ exists in $L^{2}\left(\mathbf{R}^{n}\right)$. Then $W_{1} \hat{\varphi}=0$.

Proof. Let $\psi \in \mathcal{S}\left(\mathbf{R}^{n}\right)$ with $\hat{\psi} \in \mathcal{C}_{0}^{\infty}\left(\mathbf{R}^{n} \backslash N\right)$. Let $K=\operatorname{supp} \hat{\psi}$ and write $\varphi_{+}=$ $\lim _{t \rightarrow \infty} U(0, t) U_{D}(t) \varphi$. We compute again:

$$
\begin{aligned}
i\left(U(0, t) M(t) D(t) \exp (i S(t)) \hat{\psi}-U(0, s) M(s) D(s) \exp (i S(s)) \hat{\psi}, \varphi_{+}\right) \\
=\int_{s}^{t}\left(U(0, \tau)\left(i \partial_{\tau}-H(\tau)\right) M(\tau) D(\tau) \exp (i S(\tau)) \hat{\psi}, \varphi_{+}\right) d \tau \\
=\int_{s}^{t}\left(U ( 0 , \tau ) M ( \tau ) D ( \tau ) \operatorname { e x p } ( i S ( \tau ) ) \left(\left(-V_{s}(\tau, \tau \xi)-\frac{1}{2 \tau^{2}}(\nabla S)^{2}-\frac{i}{2 \tau^{2}} \Delta S\right) \hat{\psi}\right.\right. \\
\left.\left.\quad+\frac{1}{2 \tau^{2}} \Delta \hat{\psi}+\frac{i}{2 \tau^{2}} \nabla S \cdot \nabla \hat{\psi}\right), \varphi_{+}\right) d \tau
\end{aligned}
$$

The quadratic term is written as

$$
\begin{aligned}
-\frac{1}{2 \tau^{2}}\left(U(0, \tau) M(\tau) D(\tau) \exp (i S(\tau))(\nabla S)^{2} \hat{\psi}, \varphi_{+}\right) \\
\left.=-\frac{1}{2 \tau^{2}}\left(M(\tau) D(\tau) \exp (i S(\tau))(\nabla S)^{2} \hat{\psi}, U(\tau, 0) \varphi_{+}-U_{D}(\tau) \varphi\right)\right) \\
-\frac{1}{2 \tau^{2}}\left(M(\tau) D(\tau) \exp (i S(\tau))(\nabla S)^{2} \hat{\psi}\right. \\
\left.\quad U_{0}(\tau)(1-M(-\tau)) \exp (i S(\tau,-i \nabla)) \varphi\right)-\frac{1}{2 \tau^{2}}\left((\nabla S)^{2} \hat{\psi}, \hat{\varphi}\right) .
\end{aligned}
$$

We estimate as follows:

$$
\begin{aligned}
& \left|-\frac{1}{2 \tau^{2}}\left((\nabla S)^{2} \hat{\psi}, \hat{\varphi}\right)\right| \geq \frac{1}{2} \theta_{1}(\tau)\left|\left(W_{1} \hat{\psi}, \hat{\varphi}\right)\right|-\frac{1}{2}\left(\sigma_{K}(\tau) \theta_{1}(\tau)+\rho_{K}(\tau)\right)\|\psi\|_{2}\|\varphi\|_{2} \\
& \left.\mid-\frac{1}{2 \tau^{2}}\left(M(\tau) D(\tau) \exp (i S(\tau))(\nabla S)^{2} \hat{\psi}, U(\tau, 0) \varphi_{+}-U_{D}(\tau) \varphi\right)\right) \mid \\
& \quad \leq \frac{1}{2}\left\|U(\tau, 0) \varphi_{+}-U_{D}(\tau) \varphi\right\|_{2}\left(\theta_{1}(\tau)\left\|W_{1} \hat{\psi}\right\|_{2}+\left(\sigma_{K}(\tau) \theta_{1}(\tau)+\rho_{K}(\tau)\right)\|\hat{\psi}\|_{2}\right)
\end{aligned}
$$




$$
\begin{aligned}
\left|-\frac{1}{2 \tau^{2}}\left(M(\tau) D(\tau) \exp (i S(\tau))(\nabla S)^{2} \hat{\psi}, U_{D}(\tau)(1-M(-\tau)) \exp (i S(\tau,-i \nabla)) \varphi\right)\right| \\
\leq \frac{1}{2 \tau^{2}}\|\nabla(\exp (i S(\tau))) \hat{\varphi}\|_{2}\left(\theta_{1}(\tau)\left\|W_{1} \hat{\psi}\right\|_{2}+\left(\sigma_{K}(\tau) \theta_{1}(\tau)+\rho_{K}(\tau)\right)\|\hat{\psi}\|_{2}\right) .
\end{aligned}
$$

Therefore we can treat the the quadratic term as follows: Given $\varepsilon>0$, then there exists $T(\varepsilon)>0$ such that

$$
\begin{aligned}
\mid-\frac{1}{2 \tau^{2}}(U(0, \tau) M(\tau) & \left.D(\tau) \exp (i S(\tau))(\nabla S)^{2} \hat{\psi}, \varphi_{+}\right) \mid \\
& \geq\left(\frac{1}{2}\left|\left(W_{1} \hat{\psi}, \hat{\varphi}\right)\right|-\varepsilon\right) \theta_{1}(\tau)-C(\varepsilon) \rho_{K}(\tau)
\end{aligned}
$$

for all $t>T(\varepsilon)$. The remaining terms are estimated above by an expression $\varepsilon \theta_{1}(\tau)$ plus an integrable function. Thus we can proceed as in the proof of Theorem 3.2 to conclude $W_{1} \hat{\varphi}=0$.

Example 3.11. We illustrate this result by the example

$$
V(x)=\lambda|x|^{-1 / 2}(1+\log (1+|x|))^{-\mu / 2}
$$

with $\lambda \neq 0$ and $0<\mu \leq 1$. It suffices to take $N=\{0\}, W_{1}(x)=\lambda^{2}|x|^{-3}, \theta_{1}(t)=$ $t^{-1}(\log t)^{-\mu}$, and $\rho_{K}(t)=c_{K} t^{-1}(\log t)^{-\mu-2}$. Further details are omitted.

We also give an example for the surfboard equation, see Section 4 . Note that the definition of the wave operator is changed in this case, replacing $U(0, t)$ by $U(1, t)$.

Example 3.12. Let $V(t, x)=\theta(t) \Phi\left(t^{-1} x\right)$. To verify the conditions in Assumption 3.9 we can take $\Phi \in L_{\text {loc }}^{\infty}\left(\mathbf{R}^{n}\right)$ with $\nabla \Phi \in L_{\text {loc }}^{\infty}\left(\mathbf{R}^{n}\right)$ and then take

$$
\begin{gathered}
\theta(t)=t^{-1 / 2}(\log t)^{-\mu / 2}, \quad 0<\mu \leq 1, \\
\theta_{1}(t)=t^{-1}(\log t)^{-\mu} \\
W_{1}=4(\nabla \Phi)^{2} \\
\rho(t)=C t^{-1}(\log t)^{-\mu-2} .
\end{gathered}
$$

\section{The Surfboard Schrödinger Equation}

The Schrödinger equation with a time-dependent Hamiltonian of the particular form

$$
H(t)=-\frac{1}{2} \Delta+\theta(t) \Phi\left(t^{-1} x\right)
$$

is called the surfboard Schrödinger equation. Theorem 2.3, Proposition 2.7, Theorem 3.2, and Theorem 3.10 have direct applications to this case. See the examples $2.10,3.6$, and 3.12. In this section we obtain a result on existence and unitarity of the modified wave operators for this particular equation. See [22] for previous results on this problem. 
Note that due to the singularity in the potential at $t=0$ the definition of the wave operator should be modified. We follow [22] and take

$$
\widetilde{W}_{ \pm}^{D}=\operatorname{sim}_{t \rightarrow \pm \infty} U(1, t) U_{D}(t)
$$

Another possibility would be to use $U_{D}(t-1)$ in the above definition. If $\lim _{t \rightarrow \pm \infty} \theta(t)=$ 0 , then the two definitions give the same wave operator.

Throughout this section we consider only the $t \rightarrow \infty$ case. The $t \rightarrow-\infty$ case can be treated analogously.

We introduce the notation $I=[0, \infty)$. We also use the space

$$
\Sigma(1)=\left\{\varphi \in L^{2}\left(\mathbf{R}^{n}\right) \mid\|\varphi\|_{\Sigma(1)}^{2}=\sum_{|\alpha|+|\beta| \leq 1}\left\|x^{\beta} \partial_{x}^{\alpha} \varphi\right\|_{L^{2}\left(\mathbf{R}^{n}\right)}^{2}<\infty\right\} .
$$

Assumption 4.1. Let $V(t, x)=\theta(t) \Phi\left(t^{-1} x\right)$, where $\Phi \in L^{\infty}\left(\mathbf{R}^{n}\right)$ with $\nabla \Phi \in$ $L^{\infty}\left(\mathbf{R}^{n}\right)$ and $\theta$ satisfies the following conditions:

$$
\begin{gathered}
\theta \in \mathcal{C}((0, \infty)), \quad \theta \in L^{\infty}(I) \cap L^{\alpha}(I), \quad \alpha>1, \\
\lim _{t \rightarrow \infty} t^{-1 / 2} \int_{0}^{t} \theta(\tau) d \tau=0 \\
\int_{1}^{\infty} t^{-2}\left(\int_{0}^{t}|\theta(\tau)| d \tau\right)^{2} d t<\infty
\end{gathered}
$$

We record the following result which is a special case of the results obtained in $[23]$.

Lemma 4.2 (Yajima [23]). Let $V$ satisfy Assumption 4.1. Then there exists a unique unitary propagator $U(t, s), t, s \in I$, associated with $H(t)$ given by (2.1). Furthermore, $U(t, s)$ maps $H^{2}\left(\mathbf{R}^{n}\right)$ into $H^{2}\left(\mathbf{R}^{n}\right)$ and is strongly continuous in $(t, s) \in$ $I \times I$. For $\varphi \in H^{2}\left(\mathbf{R}^{n}\right)$ we have

$$
\begin{gathered}
U(t, s) \varphi \in \mathcal{C}^{1}\left(I \times I ; L^{2}\left(\mathbf{R}^{n}\right)\right), \\
i \partial_{t} U(t, s) \varphi=H(t) U(t, s) \varphi \\
i \partial_{s} U(t, s) \varphi=-U(t, s) H(s) \varphi .
\end{gathered}
$$

This result is used without further comment in the sequel.

Lemma 4.3. Let $V$ satisfy Assumption 4.1. Let $\varphi \in \Sigma(1)$. Then $U(t, s) \varphi \in \Sigma(1)$ for all $t, s \in I$ and we have

$$
\begin{aligned}
(x+i t \nabla) U(t, s) \varphi=U(t, s)(x+i s \nabla) & \\
& +i \int_{s}^{t} \theta(\tau) U(t, \tau)(\nabla \Phi)\left(\tau^{-1}(\cdot)\right) U(\tau, s) \varphi d \tau .
\end{aligned}
$$


Proof. We start with a formal computation

$$
\begin{aligned}
i \frac{d}{d \tau}(U(s, \tau)(x+i \tau \nabla) U(\tau, s)) & =U(s, \tau)\left(\left[x+i \tau \nabla,-\frac{1}{2} \Delta+V(\tau)\right]+i \nabla\right) U(\tau, s) \\
& =U(s, \tau) i \tau \theta(\tau) \tau^{-1}(\nabla \Phi)\left(\tau^{-1}(\cdot)\right) U(\tau, s) .
\end{aligned}
$$

Integration from $s$ to $t$ and application of $U(t, s)$ on the left yields (4.5). To justify the computation one considers each component and replaces $x_{k}+i \tau \partial_{x_{k}}$ by $i \lambda\left(x_{k}+\right.$ $\left.i \tau \partial_{x_{k}}\right)\left(x_{k}+i \tau \partial_{x_{k}}+i \lambda\right)^{-1}$. After calculating the commutator above one passes to the limit $\lambda \rightarrow \infty$. Computations of this type are common in the Mourre theory, see for example [1]. Further details are omitted.

Lemma 4.4. Let $V$ satisfy Assumption 4.1. Let $\varphi, \psi \in \Sigma(1)$. Then

$$
\begin{aligned}
i \partial_{t}(\psi, U(1, t) M(t) D(t) & \exp (i S(t)) \varphi) \\
& =-\frac{1}{2 t^{2}}((x+i t \nabla) U(t, 1) \psi, M(t) D(t)(i \nabla) \exp (i S(t)) \varphi) .
\end{aligned}
$$

Proof. Assume $\varphi, \psi \in \mathcal{S}\left(\mathbf{R}^{n}\right)$. Then

$$
\begin{aligned}
i \partial_{t}(\psi, U(1, t) M(t) D(t) \exp (i S(t)) \varphi)=i \partial_{t}\left(D(t)^{-1} M(t)^{-1} U(t, 1) \psi, \exp (i S(t)) \varphi\right) \\
=\left(D(t)^{-1} M(t)^{-1} U(t, 1) \psi,\left(-\partial_{t} S(t)\right) \exp (i S(t)) \varphi\right) \\
\quad+\left(\left(-i\left[\partial_{t}, D(t)^{-1} M(t)^{-1}\right]-D(t)^{-1} M(t)^{-1} H(t)\right) U(t, 1) \psi, \exp (i S(t)) \varphi\right) .
\end{aligned}
$$

Now we use

$$
-i\left[\partial_{t}, D(t)^{-1} M(t)^{-1}\right]-D(t)^{-1} M(t)^{-1}\left(\frac{1}{2} \Delta\right)=\frac{1}{2 t^{2}} \Delta D(t)^{-1} M(t)^{-1}
$$

to continue the computation

$$
\begin{aligned}
= & \left(D(t)^{-1} M(t)^{-1} U(t, 1) \psi,\left(-V(t, t(\cdot))-\partial_{t} S(t)\right) \exp (i S(t)) \varphi\right) \\
& +\left(\frac{1}{2 t^{2}} \Delta D(t)^{-1} M(t)^{-1} U(t, 1) \psi, \exp (i S(t)) \varphi\right) .
\end{aligned}
$$

The first term vanishes due to (2.4). The second term is rewritten using

$$
(i \nabla) D(t)^{-1} M(t)^{-1}=D(t)^{-1} M(t)^{-1}(x+i t \nabla) .
$$

We get, using also the domain properties in Lemma 4.3,

$$
\begin{aligned}
& =-\frac{1}{2 t^{2}}\left(D(t)^{-1} M(t)^{-1}(x+i t \nabla) U(t, 1) \psi,(i \nabla) \exp (i S(t)) \varphi\right) \\
& =-\frac{1}{2 t^{2}}((x+i t \nabla) U(t, 1) \psi, M(t) D(t)(i \nabla) \exp (i S(t)) \varphi)
\end{aligned}
$$

We can now use Lemma 4.3 and $\nabla \Phi \in L^{\infty}\left(\mathrm{R}^{n}\right)$ to extend this result to all $\varphi, \psi \in$ $\Sigma(1)$.

Our main result is the following theorem. 
Theorem 4.5. Let $V_{\ell}=V$ satisfy Assumption 4.1. Then

$$
\widetilde{W}_{+}^{D}=\operatorname{sim}_{t \rightarrow \infty} U(1, t) U_{D}(t)
$$

exists on $L^{2}\left(\mathrm{R}^{n}\right)$ and is unitary.

Proof. Let us first prove existence. It suffices to consider $\varphi \in \mathcal{S}\left(\mathbf{R}^{n}\right)$. Let

$$
\psi(t)=U(1, t) M(t) D(t) \exp (-S(t)) \hat{\varphi} .
$$

Then for $t>s>1$

$$
\|\psi(t)-\psi(s)\|^{2}=-2 \operatorname{Re}(\psi(t)-\psi(s), \psi(s)) .
$$

By Lemma 4.3 we have $\psi(s) \in \Sigma(1)$. Thus we can write

$$
\begin{aligned}
\|\psi(t)-\psi(s)\|^{2}= & -2 \operatorname{Im} \int_{s}^{t} i \partial_{\tau}(\psi(\tau), \psi(s)) d s \\
= & \operatorname{Re} \int_{s}^{t} \tau^{-2}(M(\tau) D(\tau)(\nabla) \exp (i S(\tau)) \hat{\varphi} \\
& (x+i \tau \nabla) U(\tau, s) M(s) D(s) \exp (i S(s)) \hat{\varphi}) d \tau .
\end{aligned}
$$

Now use (4.5) and the adjoint of (4.7) to write

$$
\begin{aligned}
= & \operatorname{Re} \int_{s}^{t} \tau^{-2}(M(\tau) D(\tau)(\nabla) \exp (i S(\tau)) \hat{\varphi}, U(\tau, s) M(s) D(s)(i \nabla) \exp (i S(s)) \hat{\varphi}) d \tau \\
& +\operatorname{Re} \int_{s}^{t} \tau^{-2}(M(\tau) D(\tau)(\nabla) \exp (i S(\tau)) \hat{\varphi} \\
& \left.i \int_{2}^{\tau} \theta(\eta) U(\tau, \eta)(\nabla \Phi)\left(\eta^{-1}(\cdot)\right) U(\eta, s) M(s) D(s) \exp (-S(s)) \hat{\varphi} d \eta\right) d \tau \\
\leq & \int_{s}^{t} \tau^{-2}\|(\nabla) \exp (i S(\tau)) \hat{\varphi}\|_{2}\|(\nabla) \exp (i S(\tau)) \hat{\varphi}\|_{2} d \tau \\
& +\int_{s}^{t} \tau^{-2}\|(\nabla) \exp (i S(\tau)) \hat{\varphi}\|_{2} \int_{s}^{\tau}|\theta(\eta)| d \eta\|\nabla \Phi\|_{\infty}\|\varphi\|_{2} d \tau
\end{aligned}
$$

We have

$$
\begin{aligned}
\|(\nabla) \exp (i S(\tau)) \hat{\varphi}\|_{2} & \leq\|\nabla S(\tau)\|_{\infty}\|\varphi\|_{2}+\|\nabla \hat{\varphi}\|_{2} \\
& \leq \int_{0}^{\tau}|\theta(\eta)| d \eta\|\nabla \Phi\|_{\infty}\|\varphi\|_{2}+\|\nabla \hat{\varphi}\|_{2} .
\end{aligned}
$$

Hence the term (4.8) is estimated by

$$
c \int_{s}^{t} \tau^{-2}\left(\int_{0}^{\tau}|\theta(\eta)| d \eta+1\right)\left(\int_{0}^{s}\left|\theta\left(\eta^{\prime}\right)\right| d \eta^{\prime}+1\right) d \tau,
$$


which for $s$ large is estimated by

$$
c \int_{s}^{t} \tau^{-2}\left(\int_{0}^{\tau}|\theta(\eta)| d \eta\right)^{2} d \tau
$$

The term (4.9) is estimated by the same expression. The condition (4.4) in Assumption 4.1 implies the existence of

$$
\varphi_{+}=\lim _{t \rightarrow \infty} \psi(t)
$$

Now

$$
\begin{aligned}
\left\|U(1, t) U_{D}(t) \varphi-\varphi_{+}\right\|_{2} \leq & \left\|U(1, t)\left(U_{D}(t) \varphi-M(t) D(t) \exp (i S(t)) \hat{\varphi}\right)\right\|_{2} \\
& +\left\|U(1, t) M(t) D(t) \exp (i S(t)) \hat{\varphi}-\varphi_{+}\right\|_{2} \\
\leq & \|(1-M(-t)) \exp (i S(t,-i \nabla)) \varphi\|_{2} \\
& +\left\|U(1, t) M(t) D(t) \exp (i S(t)) \hat{\varphi}-\varphi_{+}\right\|_{2}
\end{aligned}
$$

The first term tends to zero by the computations in the proof of Theorem 2.3 and condition (4.3) in Assumption 4.1. The second term tends to zero by (4.10). Since $\mathcal{S}\left(\mathbf{R}^{n}\right)$ is dense in $L^{2}\left(\mathbf{R}^{n}\right)$, we have proved existence of $\widetilde{W}_{+}^{D}=s-\lim _{t \rightarrow \infty} U(1, t) U_{D}(t)$. To prove unitarity it suffices to prove existence of $\mathrm{s}-\lim _{t \rightarrow \infty} U_{D}(t)^{*} U(t, 1)$ on $\mathcal{S}\left(\mathbf{R}^{n}\right)$.

Let $\psi \in \mathcal{S}\left(\mathbf{R}^{n}\right)$ and let

$$
v(t)=\exp (-i S(t)) D(t)^{-1} M(t)^{-1} U(t, 1) \psi
$$

We have $v(t) \in \Sigma(1)$ by Lemma 4.3. We calculate as above for $t>s>1$

$$
\|v(t)-v(s)\|_{2}^{2}=-2 \operatorname{Im} \int_{s}^{t} i \partial_{\tau}(v(\tau), v(s)) d \tau
$$

Rewrite using Lemma 4.4

$$
\begin{aligned}
i \partial_{\tau}(v(\tau), v(s))= & -\frac{1}{2 \tau^{2}}((x+i \tau \nabla) U(\tau, 1) \psi, M(\tau) d(\tau)(i \nabla) \exp (i S(\tau)) v(s)) \\
= & -\frac{1}{2 \tau^{2}}((x+i \tau \nabla) U(\tau, 1) \psi \\
& \left.M(\tau) D(\tau)(i \nabla) \exp \left(-i \int_{s}^{\tau} \theta(\eta) d \eta \Phi\right) D(s)^{-1} M(s)^{-1} U(s, 1) \psi\right) .
\end{aligned}
$$


We commute $(i \nabla)$ to the right and then use (4.7) to get

$$
\begin{aligned}
=- & \frac{1}{2 \tau^{2}}((x+i \tau \nabla) U(\tau, 1) \psi, \\
& \left.\exp \left(-i \int_{s}^{\tau} \theta(\eta) d \eta \Phi\right) \int_{s}^{\tau} \theta(\eta) d \eta(\nabla \Phi) D(s)^{-1} M(s)^{-1} U(s, 1) \psi\right) \\
- & \frac{1}{2 \tau^{2}}((x+i \tau \nabla) U(\tau, 1) \psi, \\
& \left.\exp \left(-i \int_{s}^{\tau} \theta(\eta) d \eta \Phi\right) D(s)^{-1} M(s)^{-1}(x+i s \nabla) U(s, 1) \psi\right) .
\end{aligned}
$$

Thus we get

$$
\begin{aligned}
\|v(t)-v(s)\|_{2}^{2} \leq & \int_{s}^{t} \tau^{-2}\|(x+i \tau \nabla) U(\tau, 1) \psi\|_{2}\|\nabla \Phi\|_{\infty} \int_{s}^{\tau}|\theta(\eta)| d \eta\|\psi\|_{2} d \tau \\
& +\int_{s}^{t} \tau^{-2}\|(x+i \tau \nabla) U(\tau, 1) \psi\|_{2}\|(x+i s \nabla) U(s, 1) \psi\|_{2} d \tau
\end{aligned}
$$

Now using (4.5) we conclude that both terms are estimated by

$$
\int_{s}^{t} \tau^{-2}\left(\int_{0}^{\tau}|\theta(\eta)| d \eta\right)^{2} d \tau .
$$

Thus

$$
\psi_{+}=\lim _{t \rightarrow \infty} v(t)
$$

exists. Finally we have

$$
\begin{aligned}
& \left\|\exp (-i S(t,-i \nabla)) U_{0}(-t) U(t, 1) \psi-\check{\psi}_{+}\right\|_{2}=\left\|U(t, 1) \psi-U_{D}(t) \check{\psi}_{+}\right\|_{2} \\
& \quad \leq\left\|U(t, 1) \psi-M(t) D(t) \exp (i S(t)) \psi_{+}\right\|_{2}+\left\|M(t) D(t) \exp (i S(t)) \psi_{+}-U_{D}(t) \check{\psi}_{+}\right\|_{2} \\
& \quad \leq\left\|U(t, 1) \psi-M(t) D(t) \exp (i S(t)) \psi_{+}\right\|_{2}+\left\|(1-M(t)) \exp (i S(t,-i \nabla)) \check{\psi}_{+}\right\|_{2} .
\end{aligned}
$$

Both terms tend to zero as $t \rightarrow \infty$. This computation completes the proof.

\section{Discussion}

We discuss the results in the previous sections. The modified wave operators $W_{ \pm}^{D}$ a priori depend on the decomposition $V=V_{\ell}+V_{s}$. We have the following result which is a variant of $[6$, Theorem 3.1]. The proof is omitted.

Proposition 5.1. Assume that $U(t, s)$ is a unitary propagator. Assume $S_{j}: \mathbf{R} \times$ $\mathbf{R}^{n} \rightarrow \mathbf{R}, j=1,2$, are measurable functions. Assume

$$
W_{+}^{D, 1}=\mathrm{s}_{t \rightarrow \infty} \lim _{t \rightarrow \infty} U(0, t) U_{0}(t) \exp \left(i S_{1}(t,-i \nabla)\right)
$$


exists on $L^{2}\left(\mathrm{R}^{n}\right)$. Then

$$
W_{+}^{D, 2}=s-\lim _{t \rightarrow \infty} U(0, t) U_{0}(t) \exp \left(i S_{2}(t,-i \nabla)\right)
$$

exists on $L^{2}\left(\mathbf{R}^{n}\right)$, and $W_{+}^{D, 2}=W_{+}^{D, 1}$, if and only if for any compact $K \subset \mathbf{R}^{n}$ and any $\varepsilon>0$

$$
m e a s\left\{\xi \in K \| \exp \left(i S_{1}(t, \xi)-i S_{2}(t, \xi)\right)-1 \mid>\varepsilon\right\} \rightarrow 0 \quad \text { as } \quad t \rightarrow \infty .
$$

An analogous result holds for $W_{-}^{D, j}$ and $t \rightarrow-\infty$.

One application of this result is to prove that for certain potentials a modifier is needed. This is essentially the argument given by Dollard to prove that the ordinary wave operators do not exist for the Coulomb potential $V(x)=\lambda|x|^{-1}, \lambda \neq 0$.

The existence results obtained here generalize several known criteria by allowing the potential to be time-dependent. On the other hand, for the general time-independent potentials the strongest results on existence seem to be those in [7, Chapter XXX].

The conditions on existence in our assumptions are of a type that includes potentials for which completeness fails. As a simple example take dimension $n=4, c>0$, and $V\left(x_{1}, x_{2}, x_{3}, x_{4}\right)=-c$, for all $x$ with $\left|x_{1}\right|+\left|x_{2}\right|+\left|x_{3}\right| \leq 1$, and zero otherwise. This potential satisfies the condition in Proposition 2.5. If $c$ is chosen sufficiently large, then $\sigma_{\mathrm{ac}}(H)=\left[\lambda_{0}, \infty\right)$ for some $\lambda_{0}<0$, such that asymptotic completeness fails. This example is due to Weidmann [16]. For other examples, see [19].

Let us add a few comments on how to prove completeness in the present framework. The computations in the proof of completeness in Theorem 4.5 can be carried through in general. Let

$$
v(t)=\exp (-i S(t)) D(t)^{-1} M(t)^{-1} U(t, 0) \psi .
$$

We have for $t>s>0$

$$
\|v(t)-v(s)\|_{2}^{2}=-2 \operatorname{Im} \int_{s}^{t} i \partial_{\tau}(v(\tau), v(s)) d \tau .
$$

Repeating the computations in Section 4 we find

$$
\begin{aligned}
\left|i \partial_{\tau}(v(\tau), v(s))\right| \leq \| & V_{s}(\tau, x) U(\tau, 0) \psi\left\|_{2}\right\| \psi \|_{2} \\
& +\tau^{-2}\|(x+i \tau \nabla) U(\tau, 0) \psi\|_{2}\left\|\int_{s}^{\tau} \mu \nabla V_{\ell}\left(\mu, \frac{\mu}{s} x\right) d \mu U(s, 0) \psi\right\|_{2} \\
& +\tau^{-2}\|(x+i \tau \nabla) U(\tau, 0) \psi\|_{2}\|(x+i s \nabla) U(s, 0) \psi\|_{2}
\end{aligned}
$$

If we assume

$$
\begin{gathered}
\int_{1}^{\infty}\left\|V_{s}(\tau, x) U(\tau, 0) \psi\right\|_{2} d \tau<\infty \\
\left\|\left(\nabla V_{\ell}\right) U(t, 0) \psi\right\|_{2} \leq C(1+|t|)^{-3 / 2-\varepsilon}
\end{gathered}
$$


we can conclude existence of

$$
\lim _{t \rightarrow \infty} \exp (-i S(t)) D(t)^{-1} M(t)^{-1} U(t, 0) \psi,
$$

and if we furthermore assume

$$
\lim _{t \rightarrow \infty} t^{-1 / 2}\|\nabla S(t)\|_{\infty}=0
$$

we get existence of

$$
\lim _{t \rightarrow \infty} U_{D}(t)^{*} U(t, 0) \psi
$$

For a time-independent potential the conditions (5.1) and (5.2) would follow from decay assumptions on $V_{s}$ and $V_{\ell}$ and propagation estimates for $U(t, 0)$, together with an assumption that the spectral support of $\psi$ relative to $H$ is a positive distance away from the point spectrum of $H$. For time-dependent potentials the formulation of the concept of completeness requires some care. This problem is solved in $[10,11]$, where a geometric characterization of "scattering states" is given and asymptotic completeness is proved for a class of time-dependent long range potentials. See also the discussion in [21].

The results in Section 3 on the relation between the support of $\hat{\varphi}$ and the support of a suitably time-scaled limit of the potential are similar to those first obtained by Yajima [22] for the surfboard Schrödinger equation. Some of the results in [22] are extended in Sections 3 and 4.

The results in Section 3 give essentially sharp conditions on existence and nonexistence for potentials with a dominating term of a particular form. Without such assumptions only very weak results are obtainable. A few results of this general nature are available in the literature. In [2] necessary and sufficient conditions for the Cook criterion are discussed. One result is that the criterion

$$
\int_{1}^{\infty}\left\|\chi_{\{x|| x \mid<\alpha\}} V(t(\cdot))\right\|_{2} d t<\infty
$$

is necessary and sufficient for the Cook criterion with respect to the dense set $F^{-1} \mathfrak{C}_{0}^{\infty}\left(\mathrm{R}^{n}\right)$.

In the paper [3] necessary conditions for the existence of ordinary wave operators are obtained. The problem is considered in a fairly general framework and consequently only rather weak conditions on the potential are found. This is not surprising, since the ordinary wave operators are known to exist for oscillatory potentials with arbitrarily slow algebraic pointwise decay $O\left(|x|^{-\beta}\right), \beta>0$. As an example, take for $n=3$

$$
V(x)=\frac{\sin \left(|x|^{\alpha}\right)}{|x|^{\beta}}
$$

with $\beta>0$ and $2 \alpha+\beta>3$. For this potential the ordinary wave operators exist. Assuming this particular form of the potential White has obtained necessary and 
sufficient conditions on the parameters $\alpha$ and $\beta$ for existence (and completeness) of the ordinary wave operators, see [17]. White obtains his results essentially by combining [6, Theorem 3.1] with a proof of existence (and completeness) of modified wave operators.

Finally we discuss the results on the Stark Hamiltonians in some detail. In [8] a discrepancy between classical and quantum scattering for one-dimensional Stark Hamiltonians was discovered. It was shown that the classical wave operators exist and are complete for potentials satisfying essentially

$$
|V(x)|+\left|V^{\prime}(x)\right| \leq c(\log |x|)^{-\beta}, \quad \beta>1, \quad|x|>R .
$$

Furthermore, for potentials with behavior $V(x) \sim|x|^{-\gamma}$ as $|x| \rightarrow \infty$ the ordinary quantum mechanical wave operators do not exist, if $\gamma<1 / 2$, see $[14,8]$ and Example 3.5. Hence the discrepancy. Graf [5] (see also [18]) proposed the modifier $\exp \left(-i \int_{0}^{t} V\left(-\frac{1}{2} \tau^{2} E\right) d \tau\right)$, which is a pure phase factor. He proved existence and completeness with this modifier in any dimension, if the potential satisfies $V \in \mathfrak{C}^{1}\left(\mathbf{R}^{n}\right)$ with $\nabla V(x)=O\left(|x|^{-1-\varepsilon}\right)$ as $|x| \rightarrow \infty$. The existence part of his result is extended in Theorem 2.12, replacing the pointwise condition by an integrability condition of Enss type. In Theorem 2.13 this result is further refined. Here the pointwise requirements are essentially $\nabla_{\perp} V(x)=O\left(\left|x_{\|}\right|^{-1-\varepsilon}\right)$ and $\nabla_{\|} V(x)=O\left(\left|x_{\|}\right|^{-\frac{1}{2}-\varepsilon}\right)$ as $x_{\|} \rightarrow-\infty$. In Theorem 3.7 we then obtain a necessary condition which in Example 3.8 is used to show that the Graf modified wave operators fail to exist in dimension $n>1$, if $\nabla V(x)=O\left((|x| \log |x|)^{-1}\right)$. Thus we have found the borderline behavior for Graf's modifier in dimension $n>1$.

In [18] a different modifier is proposed. Here a modification is applied in the variable $x_{\perp}$, together with the Graf modification in the variable $x_{\|}$. The condition used allows some oscillation in the $x_{\perp}$ variable. In particular, for

$$
V(x)=\left(1+x^{2}\right)^{-\varepsilon / 2} \cos \left(\left|x_{\|}\right|^{\alpha}\right) \cos \left(\left|x_{\perp}\right|^{\beta}\right)
$$

with $\varepsilon>0,0 \leq \alpha \leq 1 / 2,0 \leq \beta \leq 1 / 2, \alpha \neq \beta$, it is shown that the ordinary wave operators exist if and only if $\varepsilon+\max \{\alpha, \beta\}>1 / 2$. White assumes for the general result on existence and completeness of his modified wave operators that the the long range part satisfies $\left|\left(\partial_{x}^{\alpha} V_{\ell}\right)(x)\right| \leq\left(1+\left|x_{||}\right|\right)^{-\varepsilon-\frac{|\alpha|}{2}}$ and $\left(\partial_{x}^{\alpha} V_{\ell}\right)(x)=o(1)$ as $|x| \rightarrow \infty$ for all multi-indices $\alpha$. This result should be compared with Theorem 2.13 which requires less differentiability and stronger decay in the $x_{\perp}$ direction, but uses the simpler Graf modifier. By the preceding discussion this condition seems close to optimal for existence.

In the one-dimensional case we first generalize the existence part of [9] to potentials satisfying essentially (5.3), i.e. the condition used in the classical mechanics result [8], see (2.27) in Proposition 2.15. The modifier used here is the one used in [9], for which the intertwining relation fails. Finally, combining Proposition 2.15 and Proposition 5.1 we show in Theorem 2.16 that this modifier can be replaced by 
Graf's modifier, thus resolving the discrepancy between the classical and quantum mechanical scattering theories for one-dimensional Stark Hamiltonians. We expect that completeness also holds with this modifier.

\section{REFERENCES}

1. H. L. Cycon, R. G. Froese, W. Krisch, and B. Simon, Schrödinger operators, Springer Verlag, Berlin, 1987.

2. M. Demuth, On necessary and sufficient conditions for the Cook-criterion, Math. Nachr. 113 (1983), 171-185.

3. M. Demuth and M. Wollenberg, Necessary conditions for the existence of wave operators, Math. Nachr. 114 (1983), 293-301.

4. J. Ginibre and T. Ozawa, Long-range scattering for non linear Schrödinger and Hartree equations in space dimension $n \geq 2$, Commun. Math. Phys. 151 (1993), 619-645.

5. G. M. Graf, A remark on long-range Stark scattering, Helv. Phys. Acta 64 (1991), 1167-1174.

6. L. Hörmander, The existence of wave operators in scattering theory, Math. Z. 145 (1976), 69-91.

7. - The analysis of linear partial differential operators, vol. IV, Springer Verlag, Berlin, 1985.

8. A. Jensen and T. Ozawa, Classical and quantum scattering for Stark Hamiltonians with slowly decaying potentials, Ann. Inst. H. Poincaré, Phys. Théor. 54 (1991), 229-243.

9. A. Jensen and $\mathrm{K}$. Yajima, On the long range scattering for Stark Hamiltonians, J. reine angew. Math. 420 (1991), 179-193.

10. H. Kitada and K. Yajima, A scattering theory for time-dependent long-range potentials, Duke Math. J. 49 (1982), 341-376.

11. _- Remarks on our paper "A scattering theory for time-dependent long-range potentials", Duke Math. J. 50 (1983), 1005-1015.

12. S. T. Kuroda, On the existence and unitarity property of the scattering operator, Nuovo Cimento 12 (1959), 431-454.

13. T. Ozawa, Long-range scattering for nonlinear Schrödinger equations in one space dimension, Commun. Math. Phys. 139 (1991), 479-493.

14. _ Non-existence of wave operators for Stark-effect Hamiltonians, Math. Z. 207 (1991), 335-339.

15. M. Reed and B. Simon, Methods of modern mathematical physics. III: Scattering theory, Academic Press, New York, 1979.

16. J. Weidmann, Spectral theory of partial differential operators, Spectral Theory and Differential Equations (W. N. Everitt, ed.), Springer Verlag, Berlin, 1975, Springer Lecture Notes in Mathematics, vol. 448, pp. 71-111.

17. D. A. W. White, Schrödinger operators with rapidly oscillating central potentials, Trans. Amer. Math. Soc. 275 (1983), 641-677.

18. _ On the long range scattering for Stark Hamiltonians, Duke Math. J. 68 (1992), 83-100.

19. D. R. Yafaev, On the break-down of completeness of wave operators in potential scattering, Commun. Math. Phys. 65 (1979), 167-179.

20. - Wave operators for the Schrödinger equation, Theoret. Math. Phys 45 (1980), 992-998.

21. __ Scattering subspaces and asymptotic completeness for the time-dependent Schrödinger equation, Math. USSR Sbornik 46 (1983), 267-283.

22. K. Yajima, The surfboard Schrödinger equation, Commun. Math. Phys. 96 (1984), 349-360.

23. __ Existence of solutions for Schrödinger evolution equations, Commun. Math. Phys. 110 (1985), 415-426. 
(A. Jensen) Department of Mathematics and Computer Science, Institute for Electronic Systems, Aalborg University, Fredrik Bajers Vej 7, DK-9220 Aalborg $\varnothing$, DENMARK

Current address: Mittag-Leffler Institute, Auravägen 17, S-182 62 Djursholm, Sweden

E-mail address, A. Jensen: jensen@ml.kva.se or matarne@iesd.auc.dk

(T. Ozawa) Department of Mathematics, Hokkaido University, Sapporo 060, Japan 